Research Paper

\title{
Deciphering the effects of PYCRI on cell function and its associated mechanism in hepatocellular carcinoma
}

\author{
Yanzhen $\mathrm{Xu}^{1,2,4 \#, \text { Wenpu Zuo }}{ }^{2,6 \#,}$ Xiao Wang2,3\#, Qinle Zhang7\#, Xiang Gan²,3, Ning Tan², Wenxian Jia ${ }^{2,3}$,

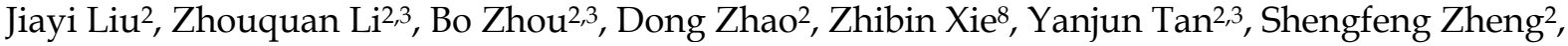 \\ Chengwu Liu ${ }^{9}$, Hongtao Li2,3, Zhijian Chen ${ }^{10}$, Xiaoli Yang $2,3 凶$, Zhaoquan Huang $1,5 \bowtie$ \\ 1. Department of pathology, Affiliated hospital of Guilin Medical University, Guilin, 541001, Guangxi, China \\ 2. Scientific Research Center, Guilin Medical University, Guilin, 541001, Guangxi, China \\ 3. Guangxi Health Commission Key Laboratory of Disease Proteomics Research, Guilin Medical University, Guilin, 541001, Guangxi, China \\ 4. Department of Pathology, Affiliated Hangzhou First People's Hospital, School of Medicine, Zhejiang University, 310000, Hangzhou, China \\ 5. Department of Pathology, the First Affiliated Hospital of Guangxi Medical University, Nanning, 530000, Guangxi, China. \\ 6. Medical Scientific Research Center, Guangxi Medical University, Nanning, 530000, Guangxi, China. \\ 7. Genetic and metabolic central laboratory, the maternal and children's health hospital of Guangxi, Nanning, 530000, Guangxi, China. \\ 8. Department of Urology, the Five Affiliated Hospital of Guangxi Medical University, Nanning, 530000, Guangxi, China. \\ 9. Department of Pathophysiology, Guangxi Medical University, Nanning, 530000, Guangxi, China. \\ 10. Department of Clinical Laboratory, the First Affiliated Hospital of Guangxi Medical University, Nanning, 530000, Guangxi, China
}

\#These authors contributed equally to this work and should be considered as co-first authors.

$\triangle$ Corresponding authors: Xiaoli Yang, Scientific Research Center, Guilin Medical University, Guilin, 541001, Guangxi, China. Guangxi Health Commission Key Laboratory of Disease Proteomics Research, Guilin Medical University, Guilin, 541001, Guangxi, China. E-mail: cncsyxl@126.com; Zhaoquan Huang, Department of pathology, Affiliated hospital of Guilin Medical University, Guilin, 541001, Guangxi, China. Department of Pathology, the First Affiliated Hospital of Guangxi Medical University, Nanning, 530000, Guangxi, China. E-mail: gxlzzq@163.com.

(c) The author(s). This is an open access article distributed under the terms of the Creative Commons Attribution License (https://creativecommons.org/licenses/by/4.0/). See http://ivyspring.com/terms for full terms and conditions.

Received: 2021.01.10; Accepted: 2021.05.16; Published: 2021.06.01

\begin{abstract}
Overexpression of pyrroline-5-carboxylate reductase 1 (PYCR1) has been associated with the development of certain cancers; however, no studies have specifically examined the role of PYCRI in hepatocellular carcinoma (HCC). Based on The Cancer Genome Atlas expression array and meta-analysis conducted using the Gene Expression Omnibus database, we determined that PYCRI was upregulated in HCC compared to adjacent nontumor tissues $(P<0.05)$. These data were verified using quantitative real-time polymerase chain reaction, western blotting, and immunohistochemistry analysis. Additionally, patients with low PYCR 1 expression showed a higher overall survival rate than patients with high PYCRI expression. Furthermore, PYCRI overexpression was associated with the female sex, higher levels of alpha-fetoprotein, advanced clinical stages (III and IV), and a younger age (< 45 years old). Silencing of PYCRI inhibited cell proliferation, invasive migration, epithelial-mesenchymal transition, and metastatic properties in HCC in vitro and in vivo. Using RNA sequencing and bioinformatics tools for data-dependent network analysis, we found binary relationships among PYCRI and its interacting proteins in defined pathway modules. These findings indicated that PYCR1 played a multifunctional role in coordinating a variety of biological pathways involved in cell communication, cell proliferation and growth, cell migration, a mitogen-activated protein kinase cascade, ion binding, etc. The structural characteristics of key pathway components and PYCR 1-interacting proteins were evaluated by molecular docking, and hotspot analysis showed that better affinities between PYCRI and its interacting molecules were associated with the presence of arginine in the binding site. Finally, a candidate regulatory microRNA, miR-2355-5p, for PYCRI mRNA was discovered in HCC. Overall, our study suggests that PYCRI plays a vital role in HCC pathogenesis and may potentially serve as a molecular target for HCC treatment.
\end{abstract}

Key words: Pyrroline-5-carboxylate reductase 1; Hepatocellular carcinoma; Antitumor; Antimetastasis; Molecular docking; RNA-seq; miRNA 


\section{Introduction}

Pyrroline-5-carboxylate reductase 1 (PYCR1), the most abundant isoform in the PYCR family, is an enzyme that catalyzes the $\operatorname{NAD}(\mathrm{P}) \mathrm{H}$-dependent conversion of $\Delta^{1}$-pyrroline-5-carboxylate (P5C) to proline [1]. Members of the human PYCR family (PYCR1-3) and proline dehydrogenase 1 (PRODH) have an established metabolic relationship, known as the proline-P5C cycle [2]. PRODH oxidizes proline to P5C, whereas proteins from the PYCR family reduce P5C back to proline [3]. The proline-P5C cycle plays a pivotal role in a myriad of cellular processes, including amino acid metabolism, and is involved in the maintenance of the intracellular redox potential and mitochondrial integrity [4]. The important regulatory contribution of PYCR1 is the catalysis of P5C to proline. Recent studies have also reported that PYCR1 converts $\Delta^{1}$-piperideine-6-carboxylate into pipecolic acid and is associated with the expression of matrix metalloproteinases [1, 4]. Furthermore, PYCR1 has demonstrated an ability to protect cells from mitochondrial fragmentation during oxidative stress [4-6]. Meanwhile, mutations in PYCR1 have been shown to induce the development of cutis laxa, a multisystem disorder characterized by premature aging, the appearance of wrinkled and lax skin, joint laxity, and a general developmental delay [7].

Hepatocellular carcinoma (HCC) is the third leading cause of cancer-related mortality worldwide and is responsible for approximately 700,000 deaths annually $[8,9]$. Many risk factors are associated with the development of HCC, among which the most critical factors include chronic hepatitis $\mathrm{C}$ or hepatitis $B$ infection, alcoholic cirrhosis, nonalcoholic steatohepatitis, and exposure to aflatoxin B1 [10-12]. Given the asymptomatic nature of HCC in the early stages, majority of HCC cases are not detected until reaching advanced stages, which results in incurable disease states [13, 14]. Moreover, patients who are diagnosed with advanced HCC are not candidates for definitive-intent therapies, such as resection, transplantation, or ablation [15]. Although first-line therapy with sorafenib is considered the standard of care for patients with advanced HCC, outcomes remain poor. As a result, the general prognosis is poor, with a 5-year overall survival (OS) rate of 3-5\% $[16,17]$. Nevertheless, recent advances have been made in the treatment of HCC, including sophisticated locoregional therapy and its associated assisted technology, newly available drugs and procedures associated with transcatheter arterial therapy, and biomarker-matched molecular-targeted therapy. Gene therapy, including gene-targeted therapy, is one of the most promising therapeutic options for HCC.
Overexpression of PYCR1 has been reported in many cancers, including non-small cell lung cancer, prostate cancer (PCa), colon cancer, and breast cancer [18-22]. Knockdown of PYCR1 was found to significantly inhibit PCa cell growth and colony formation [1], whereas PYCR1 overexpression has been correlated with poor prognoses in patients with specific cancers $[1,18]$. PYCR1 expression has also been significantly associated with breast cancer tumor size, grade, and invasiveness. It was reported that PYCR1 silencing could inhibit the expression of insulin receptor substrate 1 (IRS1) and insulin resistance via the suppression of the c-Jun $\mathrm{N}$-terminal kinase (JNK) signaling pathway, which subsequently inhibited HCC cell proliferation and promoted cell apoptosis [23]. However, it remains unclear whether PYCR1 is involved in other biological pathways related to HCC pathogenesis and development.

In the present study, we aimed to analyze the relationship between PYCR1 silencing and HCC in vitro and in vivo, in order to provide a better understanding of the mechanisms underlying HCC cell growth and survival. Furthermore, RNA sequencing (RNA-seq), bioinformatics analyses, and molecular docking were used to elucidate protein interactions that are associated with the function of PYCR1. The results will probably provide us a potential drug target for therapy or a biomarker for diagnosis and prognosis. These results will help us better understand the role of PYCR1 in the pathogenesis and development of HCC.

\section{Methods}

\section{Cells and reagents}

The HCC cell lines used in this study included Huh7 cells and LM3 cells. Because the PYCR1 expression of these two cells were higher than the other cells (Figure S1). The HCC cell lines were obtained from the China Center for Type Culture Collection (Wuhan, China). These cell lines were cultured in Dulbecco's modified Eagle's medium (DMEM; Gibco, USA) supplemented with 10\% fetal bovine serum (FBS; Gibco) and maintained in a humidified incubator with $5 \% \mathrm{CO}_{2}$ at $37^{\circ} \mathrm{C}$.

The following antibodies were used in immunoblotting and immunofluorescence studies: anti- $\beta$-actin (HRP-60008; Proteintech Group, China), anti-PYCR1 (131081; Proteintech Group), anti-Ecadherin (PA5-19479; ThermoFisher Scientific, USA), anti- $\beta$-catenin (ab32572; Abcam, USA), anti-Ncadherin (ab76011; Abcam), and anti-vimentin (ab92547; Abcam).

Reagents and materials used for the analysis of cell proliferation, migration, and invasion and for 
RNA-seq included Cell Counting Kit-8 (CCK-8; CK04, Dojindo Molecular Technologies, Inc., Rockville, MD, USA), Transwell plates ( $8 \mu \mathrm{m}$ pore size; Corning, Inc.), RNA Nano 6000 assay kit (Agilent Technologies, CA, USA), and NEB Next ${ }^{\circledR}$ Ultra $^{\mathrm{TM}}$ RNA library prep kit for Illumina ${ }^{\circledR}$ (\#E7530L; New England Biolabs, USA).

\section{Specimen collection}

A total of 106 tumor samples and adjacent nontumor liver tissues were obtained from patients who had been pathologically diagnosed with HCC and undergone a partial hepatectomy at The Affiliated Hospital of the Guilin Medical University. None of the patients received adjuvant chemotherapy or radiotherapy prior to surgery, and all cases were independently confirmed by two senior pathologists. The clinicopathological information for all cases was retrieved from the hospital's clinical database. This study was approved by the ethics committee of the Guilin Medical University, and informed consent was obtained from the patients.

\section{Meta-analysis}

$\begin{array}{ccc}\text { Seven microarray } & \text { datasets } & \text { (GSE84598, } \\ \text { GSE57957, GSE39791, GSE31370, GSE36411, }\end{array}$ GSE89377, and GSE87630) were downloaded from the Gene Expression Omnibus database (http://www. ncbi.nlm.nih.gov/geo/). Multiple probes were used for the detection of the PYCR1 gene, and the mean expression value was calculated for each probe [24]. Expression data for PYCR1 mRNA in HCC tissues were extracted from the datasets using the R software, and then a meta-analysis was performed. Standardized mean differences and 95\% confidence intervals were calculated for pooled values and indicated expression differences. Cochran's $Q$ and $I^{2}$ tests were used to evaluate heterogeneity. Based on the level of heterogeneity, two models were applied to the overall meta-analysis. A random-effect model was chosen when there was significant heterogeneity among all samples $\left(\mathrm{P}<0.05, \mathrm{I}^{2}>50 \%\right)$, and a fixed-effect model was used when there was no significant heterogeneity, as indicated by statistical analysis [25-28].

\section{Data mining using the TCGA database}

Clinical and RNA-seq data for TCGA cohorts were downloaded from the Xena Public Data Hubs (https://xena-browser.net/). Gene expression was quantified experimentally using the Illumina HiSeq 2000 RNA-seq platform. Three TCGA datasets including gene expression RNA-seq (Illumina HiSeq, $\mathrm{n}=423)$ dataset, phenotype $(\mathrm{n}=438)$ dataset, and miRNA expression RNA-seq (Illumina Hiseq, $n=420$ ) dataset were used in the study, which belongs to TCGA Liver Cancer cohort. A total of 413 samples, including 363 HCC tissues and 50 adjacent non-HCC tissues were selected for this study. Detailed patient information and PYCR1 expression levels are summarized in Table 1. The TCGA database was also employed to confirm the potential OS and relapse-free survival of patients, as well as receiver operating characteristic (ROC) signatures of PYCR1 [29].

\section{Prediction of miRNAs}

Four frequently used online tools, including miRNet (https://www.mirnet.ca), miRDB (http:// www.mirdb.org), miRTarBase (http://mirtarbase. mbc.nctu.edu.tw/php/index.php), and TargetScan (http://www.targetscan.org/vert_72/) were used to predict the upstream regulatory miRNAs of PYCR1. Only miRNAs appearing in two or more sets of the results were chosen as candidate miRNAs.

\section{Reverse transcription-polymerase chain reaction}

Total RNA was extracted using TRIzol reagent. Reverse transcription was carried out in a $20 \mu \mathrm{L}$ reaction volume with $1 \mu \mathrm{g}$ total RNA using the PrimeScript RT reagent kit (No. RR036A; TaKaRa, Japan) according to the manufacturer's instructions. Quantitative real-time polymerase chain reaction (qRT-PCR) was performed using a SYBR Green master mix (Roche, Switzerland) with a StepOne sequence detection system (Applied Biosystems). The qRT-PCR cycling conditions were as follows: initial denaturation at $95^{\circ} \mathrm{C}$ for $10 \mathrm{~min}$, followed by 40 cycles of denaturation at $95^{\circ} \mathrm{C}$ for $15 \mathrm{~s}$, annealing at $58-60{ }^{\circ} \mathrm{C}$ for $60 \mathrm{~s}$, and extension at $72{ }^{\circ} \mathrm{C}$ for $10 \mathrm{~s}$. The mRNA expression level was quantified using the $2^{-\Delta \Delta C t}$ method and normalized to that of $\beta$-actin used as an endogenous control [30]. All primers are listed in Table S1.

\section{Western blot analysis}

Proteins were extracted using RIPA lysis buffer (Solarbio Science and Technology, Beijing, China) containing a protease inhibitor cocktail (Beyotime, Shanghai, China). Protein quantification was performed using a bicinchoninic acid protein assay kit (Leagene Biotechnology, Beijing, China). Proteins were separated on $10 \%$ sodium dodecyl sulfatepolyacrylamide gels and transferred onto polyvinylidene difluoride membranes. The membranes were blocked with 5\% skim milk and then incubated with primary antibodies, including $\beta$-actin $(1: 8,000), \quad$ PYCR1 $(1: 1,000), \quad$ E-cadherin $(1: 1,000)$, $\beta$-catenin $(1: 5,000), \mathrm{N}$-cadherin (1:2,000), and vimentin $(1: 3,000)$, at $4{ }^{\circ} \mathrm{C}$ overnight. After being washed with Tris-buffered saline with Tween 20 for four times, the membranes were incubated with a horseradish peroxidase-conjugated secondary antibody at room 
temperature for $1 \mathrm{~h}$. Immunoreactive bands were visualized using the BeyoECL Plus kit (Beyotime).

\section{Immunohistochemistry}

Formalin-fixed and paraffin-embedded 4 $\mu \mathrm{m}$-thick tissue sections were deparaffinized, rehydrated, and heated with citric acid buffer for antigen retrieval for $15 \mathrm{~min}$. The samples were then placed in $0.3 \%$ hydrogen peroxide for 30 min to block endogenous peroxidase activity and treated with blocking serum for $20 \mathrm{~min}$. The slides were incubated with an anti-PYCR1 primary antibody (GTX114693; GeneTex, USA) at a 1:900 dilution at $4{ }^{\circ} \mathrm{C}$ overnight, followed by incubation with a biotinylated secondary antibody for $25 \mathrm{~min}$. The negative control slides were incubated with PBS only. The slides were rinsed in phosphate-buffered saline (PBS, $\mathrm{pH}$ 7.2), then counterstained with hematoxylin, dehydrated, and mounted in a neutral resin. The staining intensities and the proportions of positive cells in all tissues were analyzed and scored by two experienced pathologists. The results were analyzed using immunoreactivity scores based on the staining intensity and the proportion of stained cells. The staining intensity was scored as follows: $0=$ unstained; $1=$ weakly stained; 2 $=$ moderately stained; and $3=$ strongly stained. The proportion of stained cells was scored as follows: $0=$ negative; $1=1-10 \% ; 2=11-50 \% ; 3=51-80 \%$; and $4>$ $80 \%$. The intensity score was multiplied by the proportion of staining score to obtain an immunoreactivity score. A total score greater than 3 was considered positive expression; that of 1 to 3 was considered low expression; and 0 was considered negative expression.

\section{RNA interference of PYCR 1 by lentivirus}

Downregulation of PYCR1 was achieved using the following three interfering RNAs (RNAis): RNAi 1, 5'-gaGGGTCTTCACCCACTCCTA-3'; RNAi 2, 5'-tgAGAAGAAGCTGTCAGCGTT-3'; and RNAi 3, 5'-caCAGTTTCTGCTCTCAGGAA-3'. The RNAis were obtained from Shanghai GeneChemCo and were inserted between the AgeI and EcoRI sites of the GV248 vector. The short hairpin RNAs (shRNA) of GV-NC-shRNA and GV-PYCR1-shRNA were constructed as control lentivirus and shPYCR1 lentivirus for further experiment. To establish stable HCC cell lines with silenced PYCR1, $1.5 \mu \mathrm{g} / \mathrm{mL}$ puromycin was added to the culture media $48 \mathrm{~h}$ after the initiation of virus infection, and the expression of the green fluorescent protein (GFP) was observed. Cells were then trypsinized and cultured in a $100 \mathrm{~cm}^{2}$ dish to allow for expansion. The silencing of PYCR1 was confirmed by qRT-PCR and immunoblotting assays. Empty vectors were used as controls.

\section{Cell proliferation assay}

Cell proliferation was assessed using the CCK-8 solution according to the manufacturer's instructions. Briefly, Huh7 cells $\left(1.5 \times 10^{3}\right)$ from the shNC and shPYCR1 groups were seeded into a 96-well plate. After $24,48,72,96$, and $120 \mathrm{~h}$ of treatment, $10 \mu \mathrm{L}$ of the CCK-8 solution was added to each well, and incubation continued for $2 \mathrm{~h}$. The absorbance was measured at $450 \mathrm{~nm}$ using a microplate reader (BioTek Instruments, Inc., VT, USA). Each experiment was performed in triplicate.

\section{Scratch wound assay}

Huh7 and LM3 cells were seeded in a serum-free DMEM into 6-well plates $\left(7-8 \times 10^{6}\right.$ cells/well $)$ and incubated for $24 \mathrm{~h}$ to reach $80 \%$ confluence. A sterile $10 \mu \mathrm{L}$ pipette tip was then used to gently and slowly scratch the cell monolayer. Images of the wound were captured in 10 random fields using a light microscope (Olympus, Japan) at a $100 \times$ magnification. The gap width in the PYCR1-silenced cell groups was compared to that in the control using the Photoshop software at different time points. Each experiment was repeated three times.

\section{Cell invasion assay}

The in vitro invasion assay was conducted using 24-well Transwell plates with Matrigel (BD Biosciences, Franklin Lakes, NJ, USA). Cells $\left(1 \times 10^{4}\right)$ of Huh7 and LM3 were suspended in $200 \mu \mathrm{L}$ of a serum-free DMEM, seeded into the upper Transwell chamber, and cultured at $37^{\circ} \mathrm{C}$ for $24 \mathrm{~h}$, while $600 \mu \mathrm{L}$ of DMEM containing 10\% FBS was simultaneously added to the lower chamber. The invaded cells were fixed with methanol, stained with a $2.5 \%$ crystal violet stain solution (Solarbio), and then observed and photographed using an optical microscope. Five visual fields were randomly selected for quantification in each group.

\section{Flow cytometry}

Allophycocyanin-conjugated annexin $\mathrm{V}$ and 7-aminoactinomycin D (BD Biosciences, San Jose, CA, USA) were used to analyze the cell apoptosis rate. Huh7-shNC and Huh7-shPYCR1 cells were washed twice with PBS and treated with $0.25 \%$ pancreatin (without EDTA) to facilitate digestion. After adjusting the cell density to $1 \times 10^{5} / \mathrm{mL}$, the cells were incubated with fluorescent antibodies at room temperature for $25 \mathrm{~min}$, then pelleted, and resuspended. The samples were analyzed using a flow cytometer (BD Biosciences) and the FlowJo software. 


\section{In vivo tumor formation}

Male BALB/c nude mice (6-7 weeks old) were obtained from the Shanghai Institute of Materia Medica (Chinese Academy of Sciences, Shanghai, China). Cells stably transfected with the shPYCR1 or shNC $\left(1.5 \times 10^{7}\right.$ cells in $100 \mu \mathrm{L}$ of DMEM $)$ were injected subcutaneously into the upper left flank region of the mice. Tumor diameters were measured using digital calipers every 4 days. After 4 weeks of observation, the mice were sacrificed under anesthesia, and xenograft tissues were collected. All animal procedures were performed in accordance with the protocol approved by the Guilin Medical University Experimental Animal Center.

\section{RNA sequencing}

RNA purity was assessed using a K5500 spectrophotometer (Kaiao, Beijing, China), and RNA integrity and concentration were measured using the RNA Nano 6000 assay kit for the Bioanalyzer 2100 system (Agilent Technologies). A total of $2 \mu \mathrm{g}$ of RNA from each sample was used as an input material for the RNA sample preparation. Sequencing libraries were generated using the NEB Next ${ }^{\circledR}$ Ultra $^{\mathrm{TM}}$ RNA library prep kit for Illumina ${ }^{\circledR}$ according to the manufacturer's instructions. After library analysis, RNA-seq was completed using the Illumina HiSeq $X$ Ten sequencing platform, and paired-end sequence reads were obtained.

Quality control of the raw sequencing data was performed using the FastQC tool v0.11.9 ( https:// www.bioinformatics.babraham.ac.uk/). Clean RNA-seq reads were aligned to the GRCh38 human genome using HISAT v2.1.0 with default settings [31]. The aligned files were then processed using Samtools v1.9 [32], and FeatureCounts v1.6.3 was used to quantify the number of reads aligned to the coding regions of the genome [33]. Finally, DESeq2 v1.22.2 was used with the $\mathrm{R} /$ Bioconductor package to normalize read counts and identify differentially expressed genes (DEGs) [34].

\section{Bioinformatics analysis}

To analyze the functional roles of the DEGs, Gene Ontology and Kyoto Encyclopedia of Genes and Genomes pathway analyses were conducted. For proteins with multiple functions, those that are most common were assigned. Protein-protein interaction (PPI) networks were established using the STRING database.

\section{Molecular docking}

The structures of PYCR1 and its interacting proteins were downloaded from the RCSB Protein Data Bank (PDB) database (https://www.rcsb.org/).
The preparation of the PDB files, including the removal of extraneous water molecules, addition of hydrogen molecules, and adjustment of $\mathrm{pH}$-sensitive protonation, was performed using the SYBYL-X 2.0 software. Protein-protein docking was performed using the docking program HEX 8.0.0. The KFC2 (Knowledge-based FADE and Contacts) server was used to calculate protein-protein binding interface hotspots, which are defined as small fractions of residues accounting for a large fraction of the binding affinity [35]. Positive controls, interacting proteins with PYCR1 (experiment confirmed), were determined through literature review [36-40]. The average docking energy among positive control-PYCR1 complexes $\left(-1460.178 \mathrm{kcal}^{\mathrm{mol}}{ }^{-1}\right)$ was calculated to assess the docking profile in our study. Further details were recorded in Table S2.

\section{Statistical analysis}

All data were analyzed using the SPSS 17.0 software (SPSS, Chicago, IL, USA). Data are presented as the mean \pm standard deviation. One-way analysis of variance was used to compare differences between multiple groups, and Student's $t$-test was used to analyze differences between two groups. Bivariate correlations between study variables were calculated using a chi-squared test or Fisher's exact test. Survival analyses were conducted and visualized using the Kaplan-Meier method. The diagnostic ability was evaluated using an ROC curve. The sensitivity and specificity were obtained using an optimal cutoff with the max Youden index. $\mathrm{P}<0.05$ was considered statistically significant.

\section{Results}

\section{Meta-analysis of PYCR1 expression in HCC datasets}

Data were extracted from seven published and publicly available HCC datasets. Only datasets containing both HCC and their adjacent nontumor samples were used for further analysis. A meta-analysis approach was applied to these datasets as outlined in Figure 1. The results showed that PYCR1 expression was a risk factor for HCC.

\section{Data mining of the TCGA dataset}

The expression of PYCR1 mRNA was analyzed in 413 samples, including 363 HCC tissues and 50 adjacent nontumor tissues, from the TCGA dataset. Increased PYCR1 expression was found in the HCC tissues compared with the adjacent nontumor tissues (Figure 2A). Furthermore, the correlation between clinicopathological features and PYCR1 expression was investigated in 363 tumor samples. The results indicated that elevated PYCR1 expression was 
correlated with higher alpha-fetoprotein (AFP) levels, higher clinical staging (stages III and IV), and a younger age $(<45$ years old) $(p<0.05$; Table 1$)$. In addition, females were shown to have higher levels of PYCR1 than males $(P<0.05$; Table 1$)$.

We also performed ROC curve and KaplanMeier curve analyses on these TCGA samples. The area under the ROC curve was determined to be 0.650
(Figure 2B). Furthermore, as shown in Figure 2C, patients with low PYCR1 expression exhibited a higher OS rate than patients with high PYCR1 expression $(P<0.05)$. However, recurrence-free survival indicated that there was no difference in the prognosis between high and low PYCR1-expressing patients $(P>0.05$; Figure 2D).

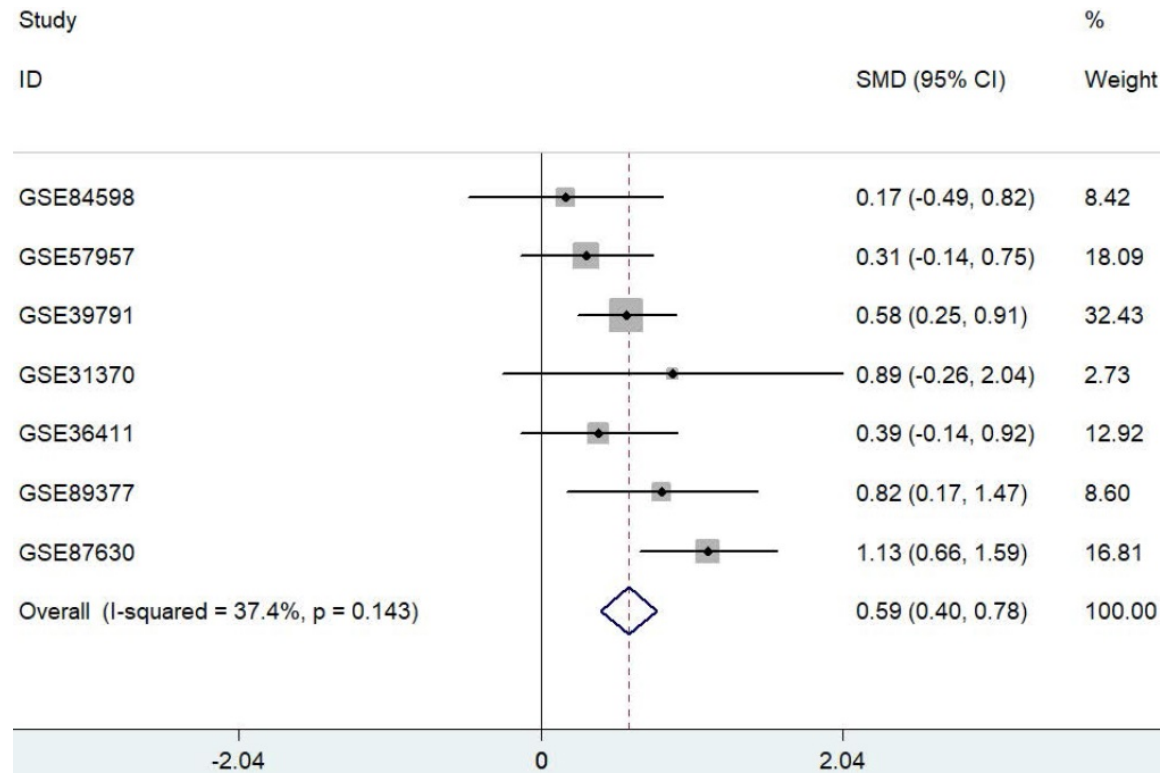

Figure 1. Forest plot depicting the association between PYCR1 mRNA expression and HCC development using a fixed-effect model. SMD: standard mean difference (tumor vs. adjacent tissue). All data were selected from GEO database.

A

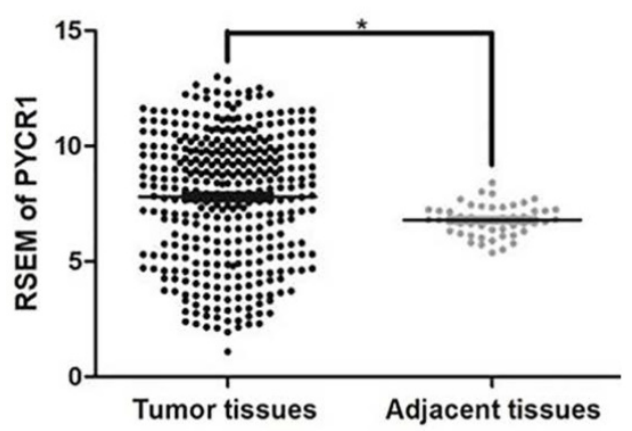

C

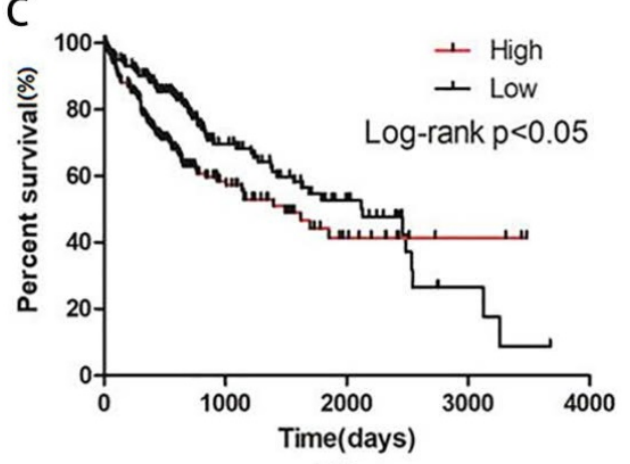

OS
B

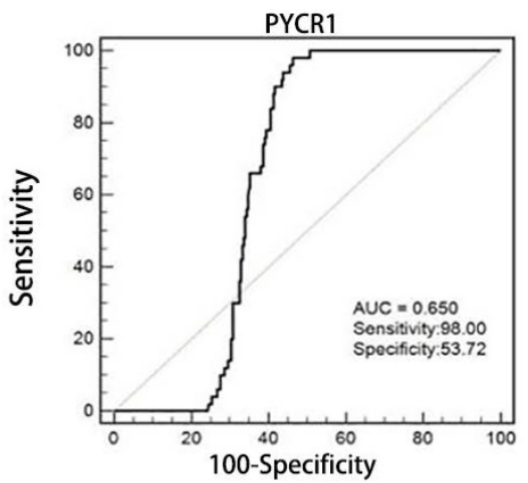

D

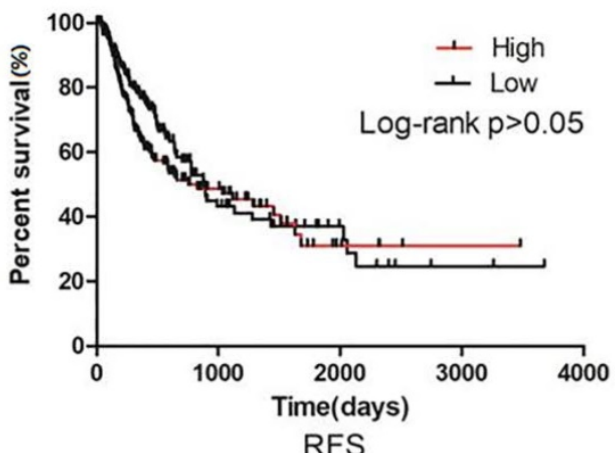

Figure 2. PYCR1 data mining in TCGA datasets. (A) RSEM of PYCR1. Significant differences were observed between tumor tissues and adjacent tissues. Student's t-tests were used to analyze the differences between two groups. (B) ROC curve for PYCR1 (**P $<0.01$ ). (C) Overall survival (OS) between patients with high and low PYCR1 expression levels. Higher PYCRI expression was associated with poorer OS $(P<0.05)$ (Kaplan-Meier analysis). (D) Recurrence-free survival (RFS) between patients with high and low PYCRI (Kaplan-Meier analysis). 

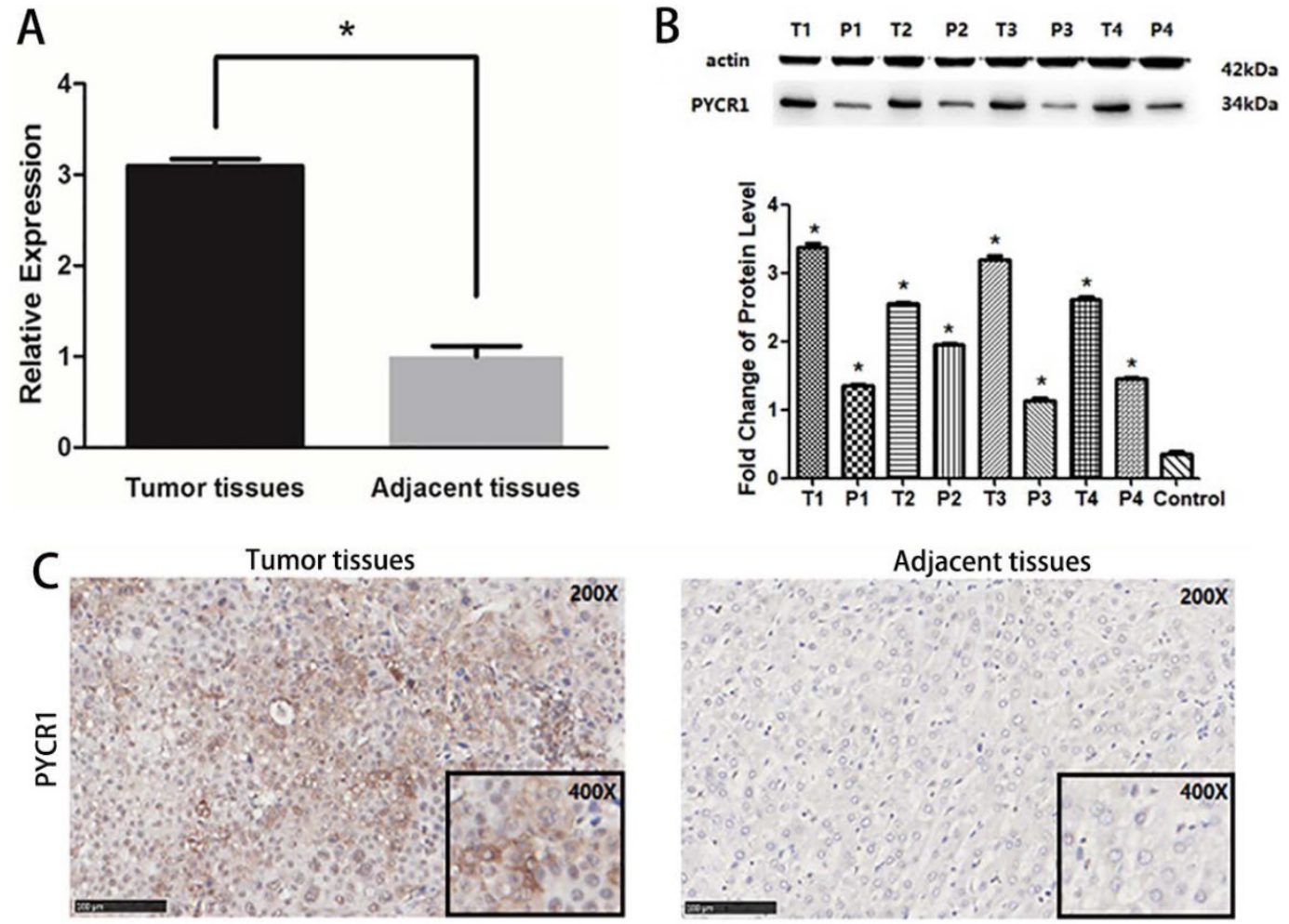

Figure 3. Expression of PYCR1 in human HCC tissues. (A) Western blot analysis of PYCR1. Lanes 1, 3, 5 , and 7: cancer tissues; lanes 2, 4, 6, and 8: adjacent tissues. (B) Relative mRNA expression of PYCR1. Tissues are listed on the $X$-axis, and the relative expression level is shown in the $Y$-axis. T: tumor; $P$ : adjacent tissues. (C) Representative images showing immunohistochemical staining of PYCRI in tumor tissues and adjacent tissues.

Table 1. Correlation between PYCRI expression and clinicopathological characteristics in HCC patients from the TCGA datasets $(n=363)$

\begin{tabular}{|c|c|c|c|}
\hline Clinical features & Case & PYCR1 level (RSEM; mean \pm SD) & $p$ value \\
\hline \multicolumn{4}{|l|}{ Sample } \\
\hline LIHC & 363 & $7.8001 \pm 2.7867$ & \multirow[t]{2}{*}{$p<0.05^{*}$} \\
\hline Adjacent tissues & 50 & $6.7891 \pm 0.6630$ & \\
\hline \multicolumn{4}{|c|}{ Age at diagnosis (years) } \\
\hline$\leq 45$ & 48 & $8.7148 \pm 2.5031$ & \multirow[t]{2}{*}{$p<0.05^{*}$} \\
\hline$>45$ & 314 & $7.6514 \pm 2.8046$ & \\
\hline Unknown & 1 & & \\
\hline \multicolumn{4}{|l|}{ Gender } \\
\hline Female & 117 & $8.3916 \pm 2.4315$ & \multirow[t]{2}{*}{$p<0.05^{*}$} \\
\hline Male & 246 & $7.5188 \pm 2.9032$ & \\
\hline \multicolumn{4}{|l|}{ The AFP in serum } \\
\hline$\leq 20 \mathrm{ng} / \mathrm{ml}$ & 143 & $6.8501 \pm 2.7379$ & \multirow[t]{3}{*}{$p<0.05^{*}$} \\
\hline$>20 \mathrm{ng} / \mathrm{ml}$ & 129 & $8.4698 \pm 2.7093$ & \\
\hline Unknown & 91 & & \\
\hline \multicolumn{4}{|l|}{ Pathologic stage } \\
\hline I-II & 251 & $7.4905 \pm 2.8598$ & \multirow[t]{3}{*}{$p<0.05^{*}$} \\
\hline III-IV & 88 & $8.6980 \pm 2.4119$ & \\
\hline Unknown & 24 & & \\
\hline \multicolumn{4}{|c|}{ Child-Pugh classification } \\
\hline A & 213 & $7.4002 \pm 2.8886$ & \multirow[t]{4}{*}{$p>0.05$} \\
\hline B & 21 & $8.1762 \pm 3.1039$ & \\
\hline $\mathrm{C}$ & 1 & & \\
\hline Unknown & 128 & & \\
\hline
\end{tabular}

TCGA: The Cancer Genome Atlas; LIHC: Liver Hepatocellular Carcinoma; HCC: hepatocellular carcinoma; RSEM: RNA-Seq by Expectation-Maximization; PYCR1: Pyrroline-5-carboxylate reductase 1; AFP: Alpha Fetoprotein; SD: standard deviation. ${ }^{*} p<0.05$.

\section{Verification of PYCRI expression in HCC tissues and relationship between PYCRI expression and HCC clinicopathological features}

PYCR1 mRNA expression was found to be significantly higher in tumor tissues than in adjacent nontumor tissues in 106 paired tumor and adjacent tissues sets $(P<0.05$; Figure 3A). Further, the PYCR1 protein expression was consistent with that of mRNA (Figure 3B and 3C). Higher expression of PYCR1 was also associated with a younger age $(<45$ years old $)$ and higher clinical staging (stages III and IV). However, no association was detected between PYCR1 expression and other examined clinical and demographic features, including sex, tobacco smoking, alcohol consumption, hepatitis B surface antigen levels, AFP levels, alanine aminotransferase (ALT) levels, and tumor size (Table 2).

\section{Downregulation of PYCRI inhibits cell proliferation, migration, invasion, and tumor growth}

To assess the effects of PYCR1 in HCC, we established PYCR1 interference by infecting with control lentivirus and shPYCR1 lentivirus in HCC cells. We detected the PYCR1 expression in HCC cell lines by Western blot. And we found the PYCR1 expression of Huh7 was higher than others in 
non-metastatic cell lines. Additionally, PYCR1 expression in LM3 cells was the highest among three high-metastatic cell lines. Taken together, Huh7 and LM3 cell lines, which have comparably high expressions of PYCR1 among non-metastatic and metastatic cells respectively, were chosen to conduct further target gene silencing experiments (Figure S1). Compared with controls, infected with shPYCR1 lentivirus led to suppressed PYCR1 expression in both
mRNA and protein levels. The transcription of PYCR1 was suppressed dramatically by shPYCR1(1-3) compared with shNC in Huh7 (Figure 4A and 4B). Significantly, we found shPYCR1(3) could more efficiently suppress the expression of PYCR1 than others. And shPYCR1(3) obviously suppressed the expression of PYCR1 mRNA and protein in LM3 (Figure $4 \mathrm{~A}$ and $4 \mathrm{~B}$ ).
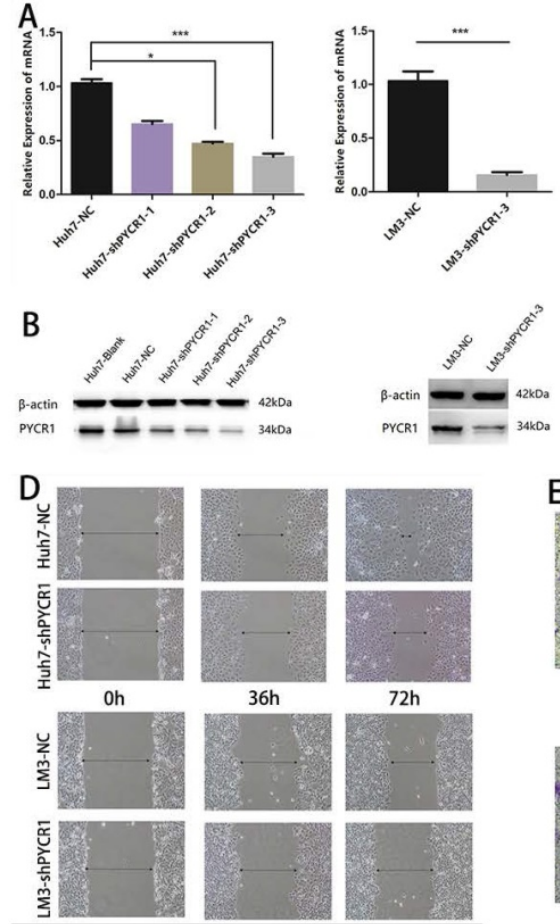

C
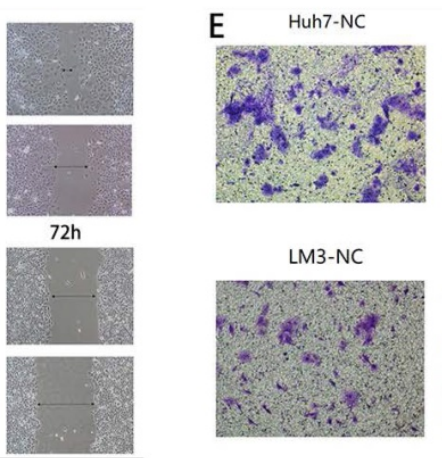

G
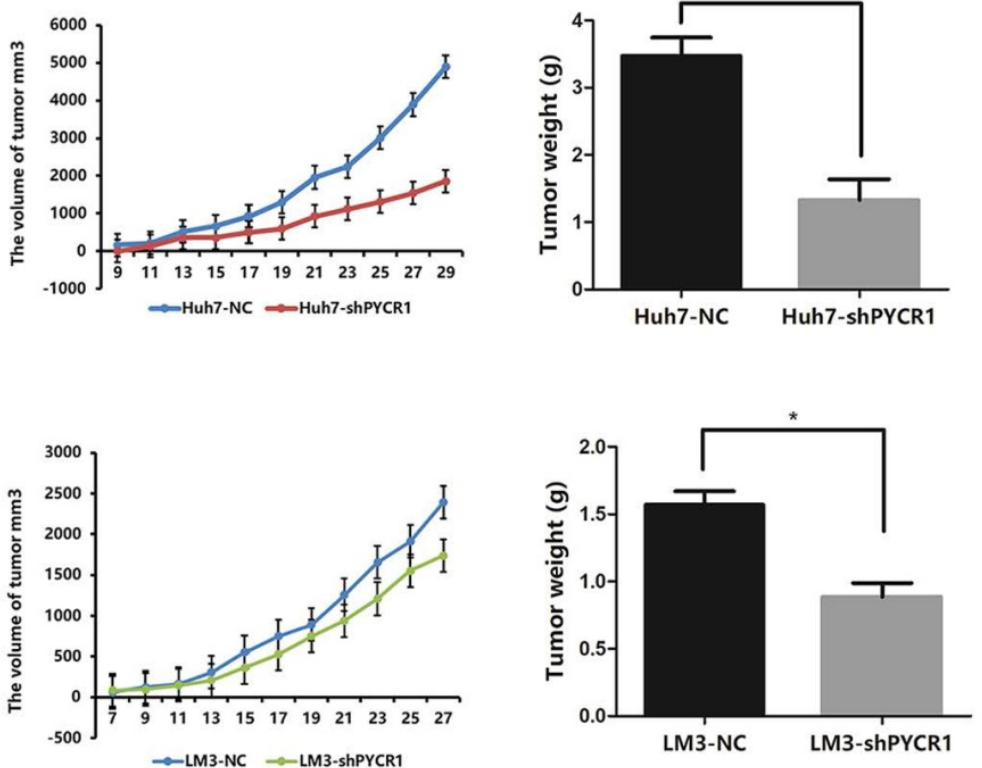
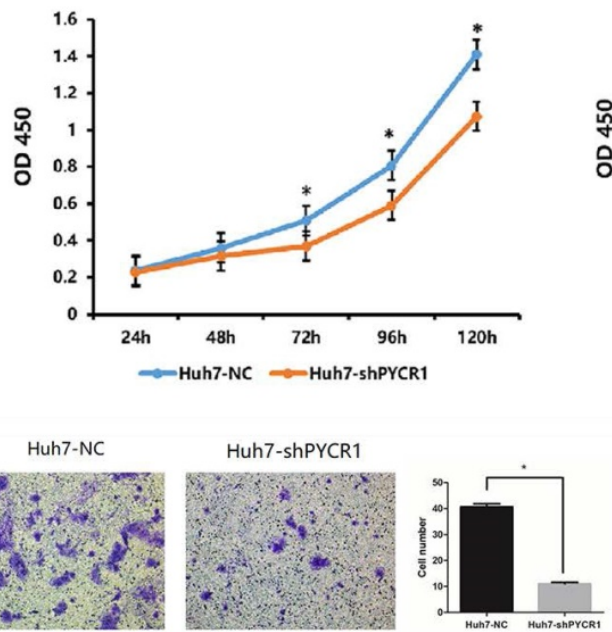

LM3-NC

LM3-shPYCR1
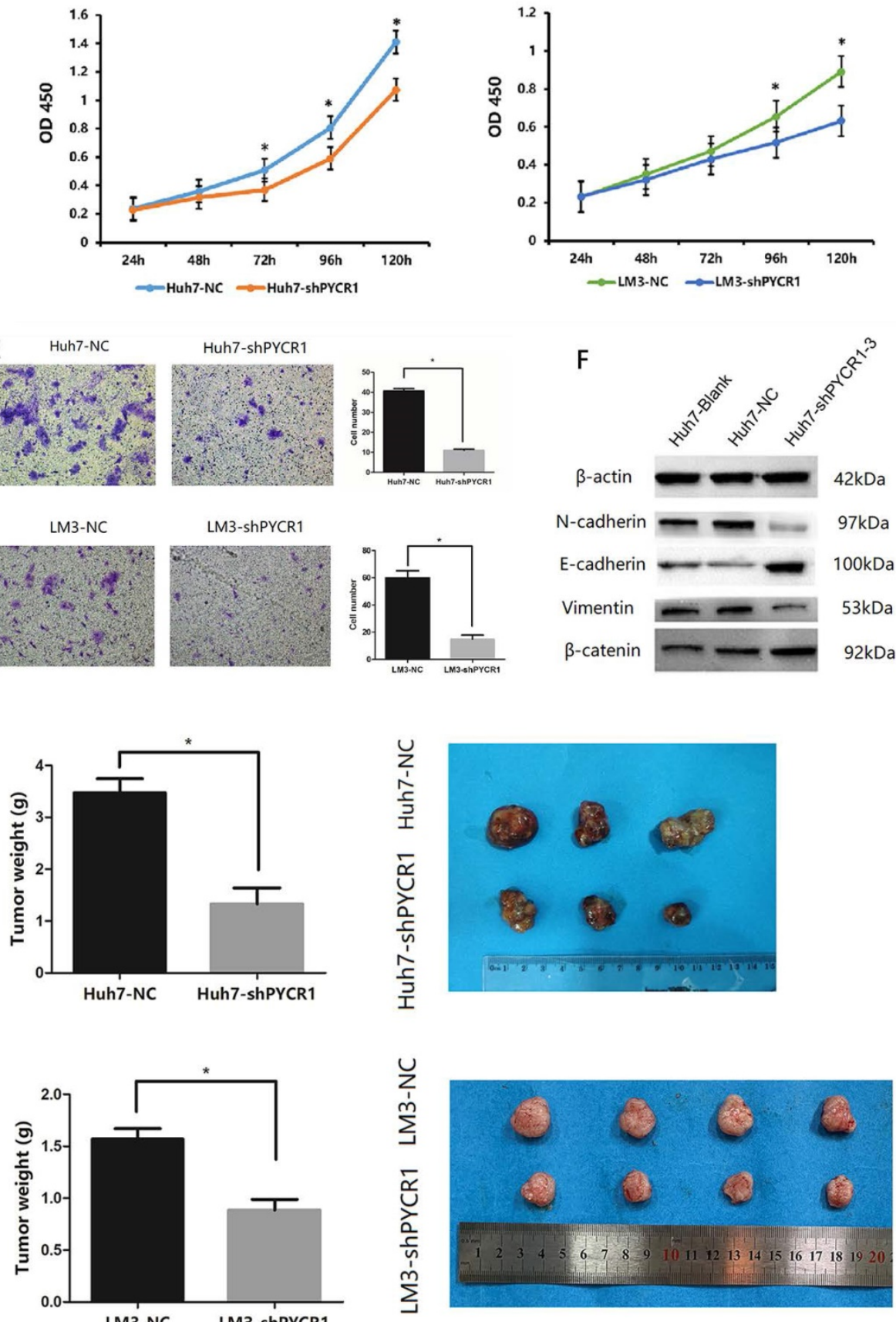

Figure 4. Knockdown of PYCRI inhibits proliferation, migration, invasion, EMT and tumor growth capacities of HCC cells. (A-B) Western blot analysis and qRT-PCR were used to detect PYCR1 expression in Huh7 and LM3 cell lines transfected with three kinds of PYCR1 RNAi. (C) The CCK8 analysis revealed down-regulation of PYCR1 caused inhibition of cellular proliferation. (D) Scratch-wound assay showed that down-regulation of PYCR1 in Huh7 cells inhibited cell migration. (E) Down-regulation of PYCR1 reduced the invasiveness of HCC cells (data are shown as averages $\pm S D, n=5, * P<0.05$ ). (F) Western blot analysis of EMT-related proteins showing the expression of E-cadherin, $\mathrm{N}$-cadherin, vimentin, and $\beta$-catenin as quantified by western blot. (G) Down-regulation of PYCR1 in Huh7 cells inhibited tumor growth in nude mice $(* P<0.05)$. 
Table 2. Correlation between the factors and clinicopathological characteristics in hepatocellular carcinoma $(n=106)$

\begin{tabular}{|c|c|c|c|c|}
\hline \multirow[t]{2}{*}{ Clinical features } & \multirow{2}{*}{$\begin{array}{l}\text { Case } \\
\text { number }\end{array}$} & \multicolumn{2}{|l|}{ PYCR1 } & \multirow{2}{*}{$P$ value } \\
\hline & & Low $/ \operatorname{Neg}(n=60)$ & $\operatorname{High}(n=46)$ & \\
\hline \multicolumn{5}{|l|}{ Age (years) } \\
\hline$\leq 45$ & 47 & 21 & 26 & $0.032<0.05^{*}$ \\
\hline$>45$ & 59 & 39 & 20 & \\
\hline \multicolumn{5}{|l|}{ Gender } \\
\hline Male & 84 & 45 & 39 & $0.239>0.05$ \\
\hline Female & 22 & 15 & 7 & \\
\hline \multicolumn{5}{|l|}{ Smoking } \\
\hline Yes & 44 & 21 & 23 & $0.164>0.05$ \\
\hline No & 62 & 39 & 23 & \\
\hline \multicolumn{5}{|l|}{ Drinking } \\
\hline Yes & 44 & 25 & 19 & $1.000>0.05$ \\
\hline No & 62 & 35 & 27 & \\
\hline \multicolumn{5}{|l|}{ HBsAg infection } \\
\hline Yes & 83 & 50 & 33 & $0.163>0.05$ \\
\hline No & 23 & 10 & 13 & \\
\hline \multicolumn{5}{|l|}{$\operatorname{AFP}(n g / m L)$} \\
\hline$\leq 20$ & 32 & 19 & 13 & $0.832>0.05$ \\
\hline$>20$ & 74 & 41 & 33 & \\
\hline \multicolumn{5}{|l|}{ ALT (U/L) } \\
\hline$\leq 40$ & 61 & 35 & 26 & $1.000>0.05$ \\
\hline$>40$ & 45 & 25 & 20 & \\
\hline \multicolumn{5}{|l|}{ AST (U/L) } \\
\hline$\leq 40$ & 53 & 27 & 26 & $0.327>0.05$ \\
\hline$>40$ & 53 & 33 & 20 & \\
\hline \multicolumn{5}{|l|}{ Clinical staging } \\
\hline $\mathrm{I}+\mathrm{II}$ & 58 & 39 & 19 & $0.019<0.05^{*}$ \\
\hline III + IV & 48 & 21 & 27 & \\
\hline \multicolumn{5}{|l|}{ Tumor size (cm) } \\
\hline$\leq 5$ & 43 & 25 & 18 & $0.843>0.05$ \\
\hline$>5$ & 63 & 35 & 28 & \\
\hline \multicolumn{5}{|l|}{ Metastasis } \\
\hline Yes & 15 & 6 & 9 & $0.174>0.05$ \\
\hline No & 91 & 54 & 37 & \\
\hline \multicolumn{5}{|l|}{ Invasion (DHCC) } \\
\hline Yes & 50 & 27 & 23 & $1.000>0.05$ \\
\hline No & 44 & 24 & 20 & \\
\hline Unknown & 12 & 9 & 3 & \\
\hline \multicolumn{5}{|l|}{ Lack of P53 } \\
\hline Yes & 70 & 37 & 33 & $0.274>0.05$ \\
\hline No & 29 & 19 & 10 & \\
\hline Unknown & 7 & 4 & 3 & \\
\hline \multicolumn{5}{|l|}{ Sample } \\
\hline $\mathrm{HCC}$ & 106 & 60 & 46 & $0.021<0.05^{*}$ \\
\hline Adjacent tissue & 106 & 77 & 29 & \\
\hline
\end{tabular}

AFP: Alpha Fetoprotein; DHCC: diffuse hepatocellular carcinoma; Neg: negative; NS: no significance; ${ }^{*}$ : $p<0.05$.

Downregulation of PYCR1 expression was found to inhibit cell proliferation (Figure 4C). The migration capacity and invasiveness were assessed using Transwell plates and a wound scratch assay. As shown in Figure $4 \mathrm{D}$ and $4 \mathrm{E}$, downregulation of PYCR1 expression significantly inhibited the migration and invasiveness of Huh7 and LM3 cells in vitro. However, no significant differences were observed in the levels of apoptosis between the experimental and control groups (data not shown). Furthermore, we quantified the expression levels of proteins associated with epithelial-mesenchymal transition (EMT) and determined whether their expression was affected by downregulation of PYCR1. Our results suggested that the expression of E-cadherin and $\beta$-catenin was significantly increased in cells with downregulated PYCR1 expression, whereas that of $\mathrm{N}$-cadherin and vimentin was decreased (Figure 4F).

We further analyzed the role of PYCR1 in vivo using an immunodeficient nude mouse model. Compared with the control mice, those with downregulated PYCR1 expression exhibited a delayed onset of tumor growth and a smaller tumor volume after 4 weeks of observation (Figure 4G).

\section{Global profiling of differentially expressed mRNAs}

Global mRNA profiling was performed in Huh7 cells with either normal or downregulated expression levels of PYCR1. Genes were categorized as up- or downregulated when their expression fold changes were $>1.5$ or $<0.67$ compared to the control group, respectively $(P<0.05)$. Based on these parameters, 569 genes were found. After removing noncoding genes, 425 DEGs were identified, of which 298 were upregulated and 127 were downregulated following PYCR1 silencing (Figure 5A-D). Some of these genes encoded proteins that were known to interact with PYCR1; however, most of the genes were identified for the first time (Figure 5E).

\section{Data-dependent network analysis of PYCR 1-interacting proteins in HCC cells}

As shown in Figure 6A, each DEG was assigned to a specific functional category, including cell communication, cell proliferation and growth, cell migration, a mitogen-activated protein kinase (MAPK) cascade, ion binding, immune stimulus, cell component, transport, and catalytic activity. PPI analysis further demonstrated that many of the proteins were associated with each other (Figure 6B).

\section{Validation of the HCC-specific PYCRI interactome dataset}

In our selected pool of PYCR1-interacting proteins, more attention was paid to the genes known to be involved in vital pathway modules, such as cell communication, cell proliferation and growth, cell migration, an MAPK cascade, and ion binding. Based on their large fold changes and biological functional analysis, some of the DEGs were further confirmed by qRT-PCR, and the results were consistent with those of RNA-seq (Figure 6C).

\section{Molecular docking}

The structures of eight interacting proteins, which were associated with key pathway modules, such as MAPK pathways, cell migration, cell 
communication, and cell proliferation/growth, and identified downstream of PYCR1, were found in the PDB database. The information obtained from the PDB files and HEX docking results is shown in Table 3 . Total energy values ( $\left.E_{\text {total }}\right)$ for PYCR1 and its eight target proteins were determined to be as follows: apoptogenic protein 1 (APOP1; $-436.7 \mathrm{kcal} \mathrm{mol}^{-1}$ ), glutamate ionotropic receptor kainite type subunit 2 (GRIK2; $-513.79 \mathrm{kcal} \mathrm{mol}^{-1}$ ), RhoGD12 (ARHGDIB; $-820.41 \mathrm{kcal} \mathrm{mol}^{-1}$ ), dual specificity phosphatase 9 (DUSP9; -906.94 kcal $\mathrm{mol}^{-1}$ ), chorionic gonadotropin subunit alpha (CGA; $-1,488.78 \mathrm{kcal} \mathrm{mol}^{-1}$ ), retinol-binding protein 4 (RBP4; $\left.-1,656.65 \mathrm{kcal} \mathrm{mol}^{-1}\right)$, nuclear receptor subfamily 1 group $\mathrm{H}$ member 4

A

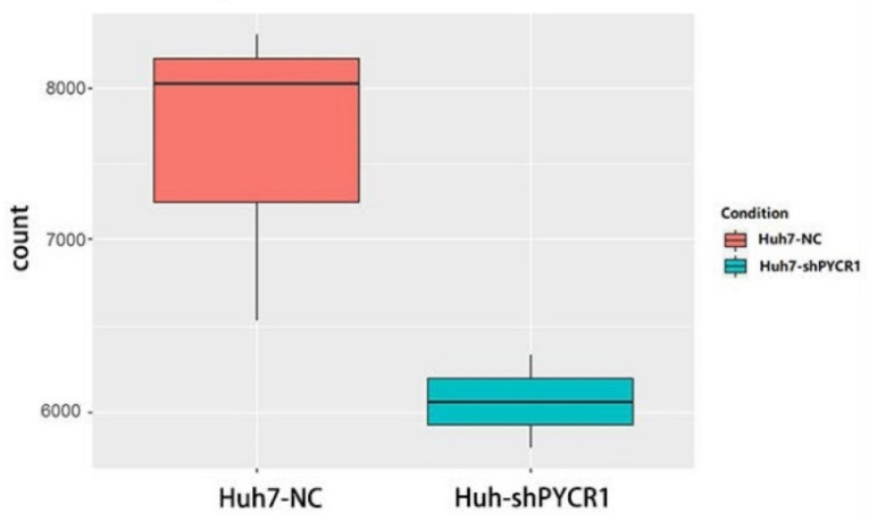

C Volcano plot

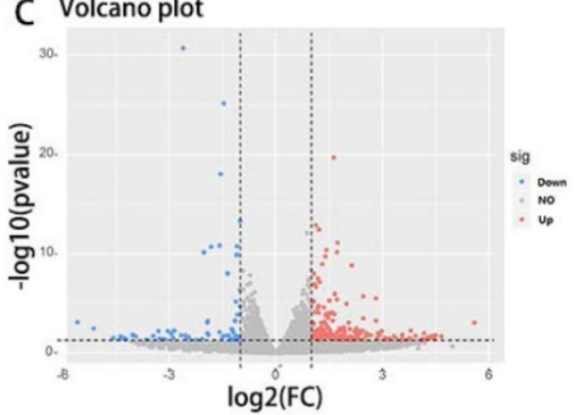

$\mathrm{D}$

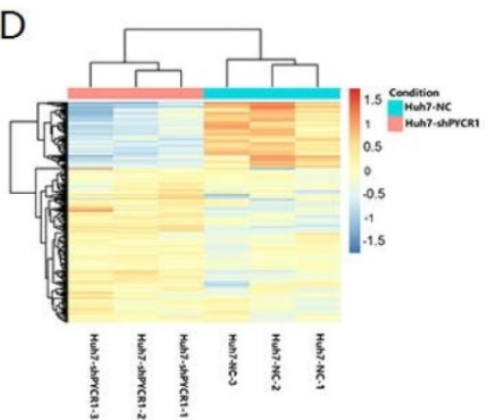

(NR1H4; $-1,659.98 \mathrm{kcal} \mathrm{mol}^{-1}$ ), and serpin family E member 1 (SERPINE1; $-1,889.79 \mathrm{kcal} \mathrm{mol}^{-1}$ ). The complexes generated during docking are shown in Figure 7, and the $E_{\text {total }}$ values reflect the possibility of binding, with a lower value correlating with easier and stronger binding to other proteins [35]. The results of the hotspot analysis are summarized in Figure 7. We found that better affinities between PYCR1 and its interacting molecules were associated with the presence of arginine (Arg) in the binding site. The diagnostic value of PYCR1, combined with its interacting proteins, was further tested based on the RNA-seq data from TCGA by binary logistic regression (Figure S2 and Table S3).
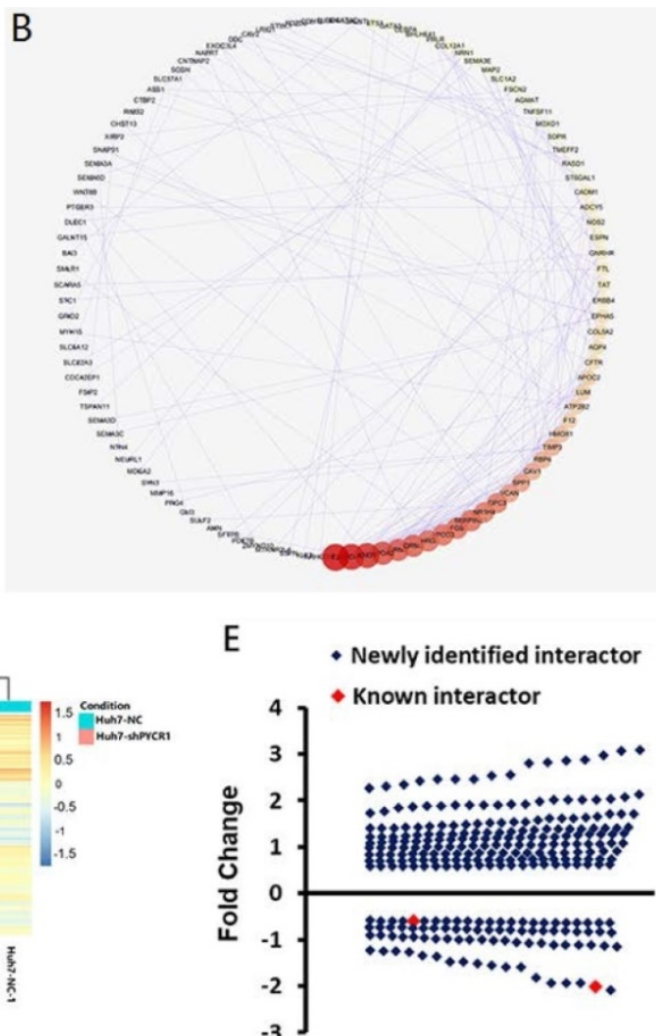

Figure 5. Down-regulation of PYCR1 resulted in differential mRNA expression, as demonstrated by RNA-seq analysis. (A) The PYCR1 mRNA expression level between PYCR1-silenced Huh7 cells and Huh7 cell control cells as determined by RNA-seq analysis. The gene counts were obtained by quantifying the number of reads that aligned with PYCR1 using the feature Counts programs. (B) The protein-protein interaction networks associated with the differentially expressed genes from RNA-Seq analysis. The interaction network is presented as a Cytoscape degree sorted circle summary layout; the nodes are shown as circles and the edges are shown as lines linking two nodes. (C) Volcano plot. Blue spots: up-regulated genes; red spots: down-regulated genes. (D) Heatmap. Significantly differentially expressed genes observed between control Huh7 cells and PYCR1-silenced Huh7 cells. (E) Distribution of up/down-regulated genes after the silencing of PYCR1 in HCC cells. Red spots: known genes; blue spots: newly identified genes.

Table 3. Molecular docking total energy values for PYCRI and its interactor proteins with hot spot analysis

\begin{tabular}{|c|c|c|c|c|c|}
\hline Target proteins & Name of PDB files & Positions & $\mathrm{E}_{\text {total }} \mathrm{kcal} \mathrm{mol}^{-1}$ & Hot spots of PYCR1 & Hot spots of target protein \\
\hline APOA1 & $3 \mathrm{~K} 2 \mathrm{~S}$ & $25-267$ & -436.7 & / & / \\
\hline GRIK2 & $3 \mathrm{QXM}$ & $\begin{array}{l}\text { 429-544 } \\
667-806\end{array}$ & -513.79 & / & / \\
\hline ARHGDIB & 1DS6 & 23-199 & -820.41 & / & / \\
\hline DUSP9 & $2 \mathrm{HXP}$ & 201-345 & -906.94 & ARG200(A), GLU221(B) & HIS251(G), GLU278(G), GLN282(G) \\
\hline CGA & 1HD4 & $25-116$ & -1488.78 & $\begin{array}{l}\text { ASP165(A), ARG264(A), ARG266(A), } \\
\text { GLU267(A), GLN269(A), SER270(A), MET271(A), } \\
\text { ILE263(C) }\end{array}$ & $\begin{array}{l}\text { LEU41(G), ARG42(G), SER43(G), VAL61(G), } \\
\text { LYS63(G), TYR65(G), TYR88(G), TYR89(G), } \\
\text { SER92(G) }\end{array}$ \\
\hline RBP4 & $5 \mathrm{NU} 7$ & $19-200$ & -1656.65 & / & / \\
\hline NR1H4 & 4QE6 & $258-486$ & -1659.98 & / & / \\
\hline SERPINE1 & $1 \mathrm{C} 5 \mathrm{G}$ & $1-402$ & -1889.79 & SER43(B), ARG46(B) & LEU211(G), THR228(G), ASN229(G) \\
\hline
\end{tabular}



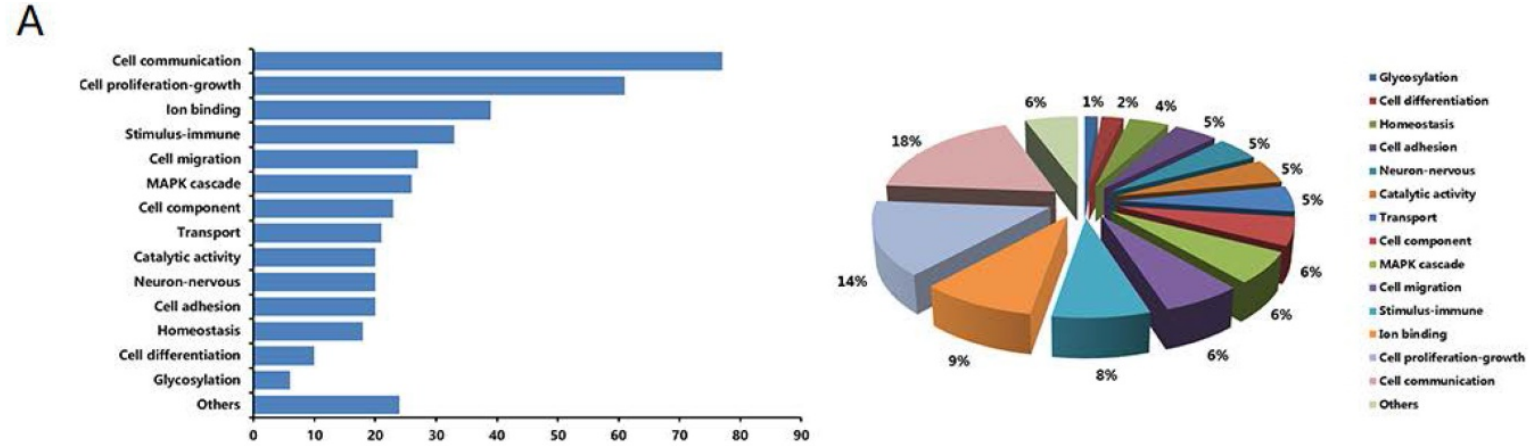

B

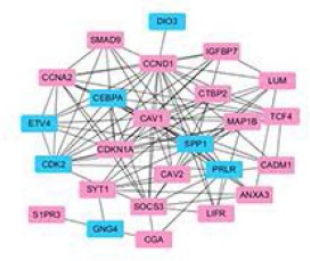

Cell Proliferation-growth

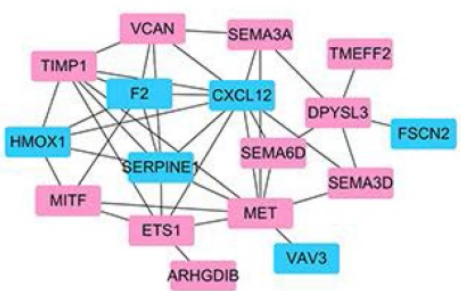

Cell Migration

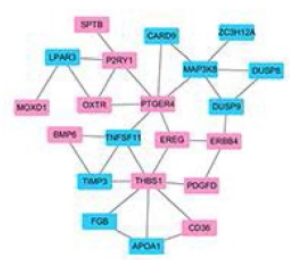

MAPK cascade

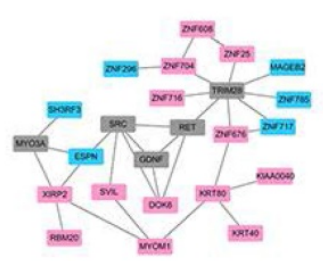

Ion binding

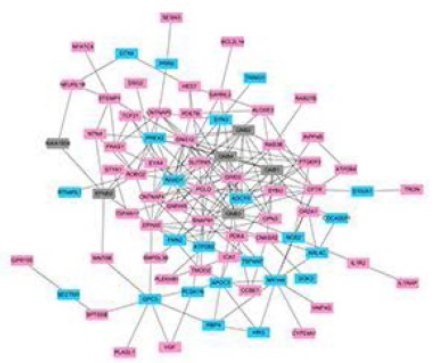

Cell Communication

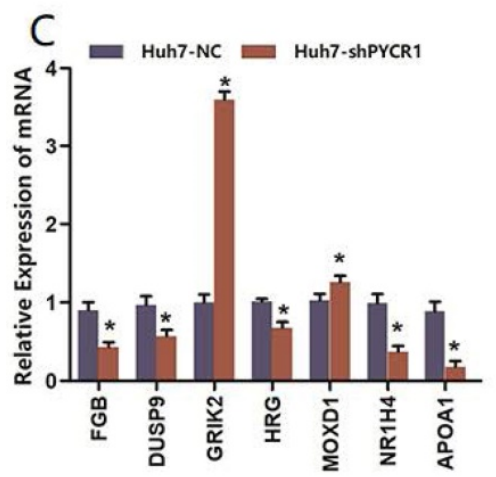

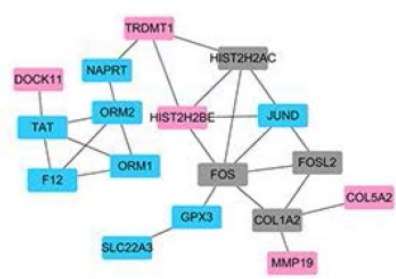

Stimulus- immune
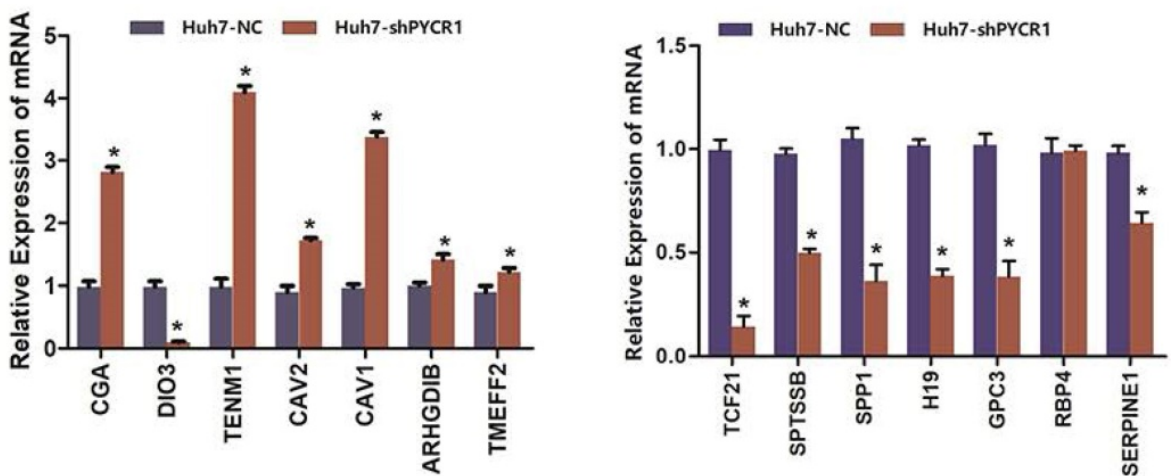

Figure 6. Biological functional groups of differentially expressed genes in HCC cells. (A) Multiple functional categories. (B) Pathway modules. Red spot: up-regulated genes after silencing of PYCR1; blue spot: down-regulated genes after silencing of PYCR1; gray spot: potential node genes. (C) Validation of mRNA levels of differentially expressed genes obtained via RNA-seq with qRT-PCR.

miR-2355-5p might be an upstream regulator for PYCRI mRNA in HCC

All predicted miRNAs from the four miRNA databases are listed in Table S4. According to the quantitative Venn diagram (Figure 8A), five candidates, including miR-1253, miR-6081, miR-3150a-3p, miR-2355-5p, and miR-5000-3p, were determined to potentially target PYCR1 mRNA. Based on the TCGA RNA-seq data, only miR-2355-5p was differentially expressed between HCC tissues and adjacent nontumor tissues and was downregulated in HCC samples compared to adjacent nontumor samples (Figure 8B). The expression levels of miR-3150a-3p and miR-5000-3p were similar in HCC and adjacent nontumor samples, while no data 
pertaining to miR-1253 and miR-6081 were available in the TCGA database. The validation using qRT-PCR showed that miR-2355-5p expression was decreased in HCC tissues compared to adjacent nontumor tissues (Figure 8C), with a negative correlation between PYCR1 mRNA and miR-2355-5p expression (Figure 8D). After silencing PYCR1, miR-2355-5p was found to be upregulated in HCC cell lines (Figure $8 \mathrm{E}$ and $8 \mathrm{~F}$ ).

\section{Discussion}

PYCR1 plays an important role in the normal physiological functions of cells and in the development of various diseases [41, 42]. In particular, previous studies have shown that PYCR1 is closely associated with the development of various cancers, including $\mathrm{PCa}$, lymphoma, and breast cancer $[1,18,43]$. However, the specific mechanism responsible for the role of PYCR1 in cancer development and progression has not been adequately elucidated. Our findings suggest that PYCR1 is involved in HCC pathogenesis and may thus serve as a diagnostic and therapeutic target for

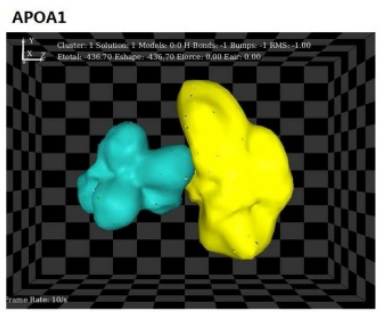

ARHGDIB
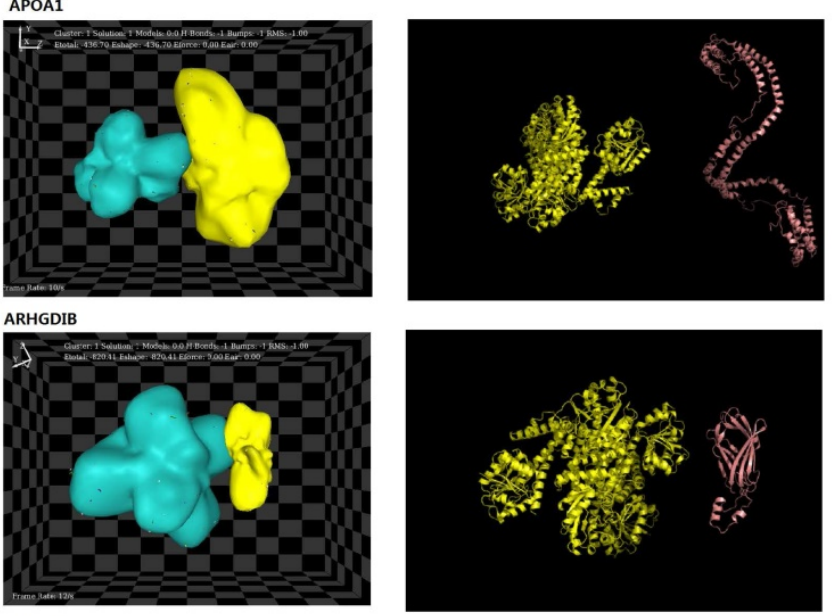

NR1H4
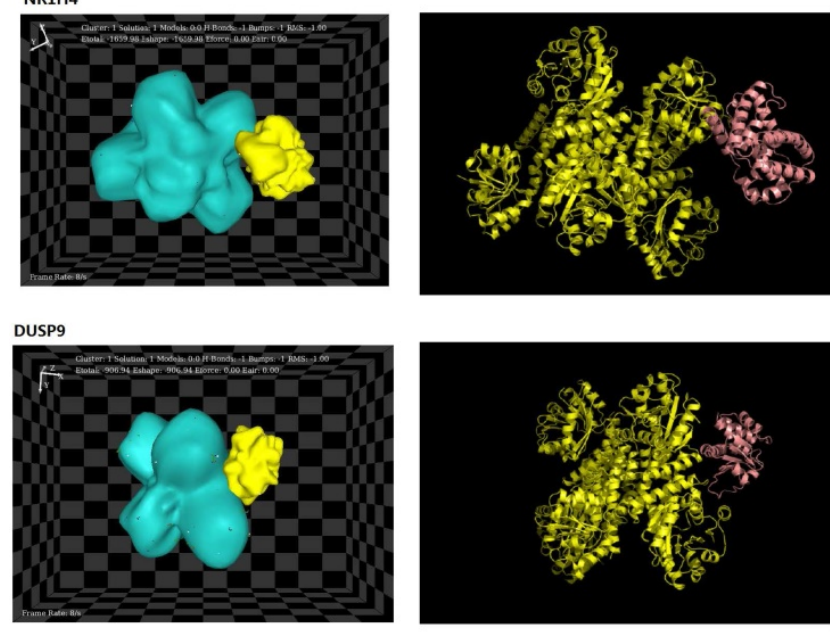

Figure 7. Docking results of PYCR1 and its target proteins including APOP1, GRIK2, ARHGDIB, DUSP9, CGA, RBP4, NRI H4, and SERPINE1. PDB files for each target protein and PYCRI were collected from https://www.rcsb.org/.
HCC management.

In the present study, PYCR1 mRNA and protein expression levels were found to be higher in HCC tissues than in adjacent nontumor tissues. Analysis of PYCR1 expression in patients from the TCGA cohort revealed that the upregulation of PYCR1 correlated with sex, elevated AFP levels, higher clinical staging (stages III and IV), and a younger age ( $<45$ years old). Furthermore, PYCR1 upregulation was found to be associated with a poor OS. These results suggest that PYCR1 expression may be an effective predictive factor for OS in patients with HCC. Similar correlations between PYCR1 expression and clinical features (age and clinical staging) were also observed in our patients with HCC; however, there was no association between PYCR1 expression and AFP level or sex. This discrepancy may be due to a largely Caucasian population in the TCGA database, whereas our HCC tissues were exclusively obtained from Chinese patients. Thus, ethnic diversity may impact the effect of PYCR1 on HCC development. Additional HCC samples from patients of different ethnicities should be included in further studies.
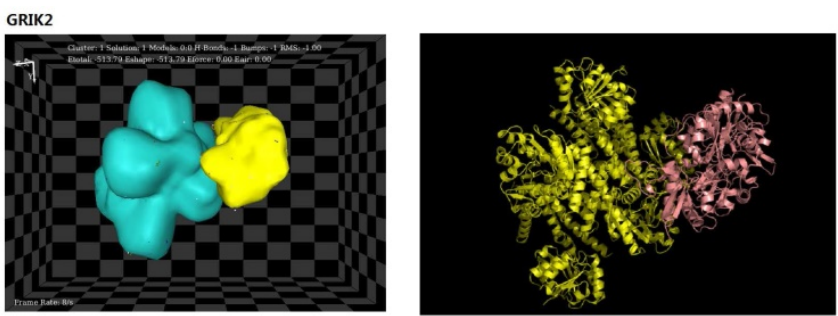

SERPINE1
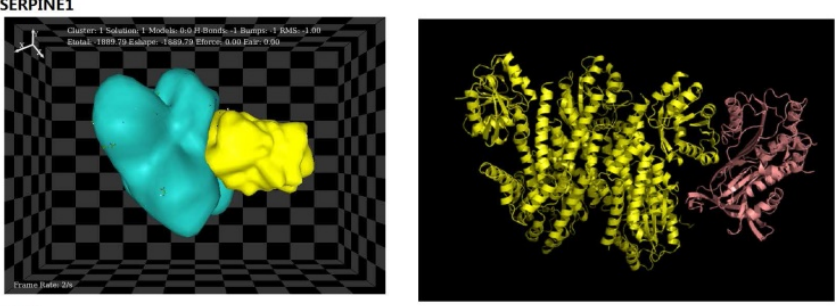

RBP4
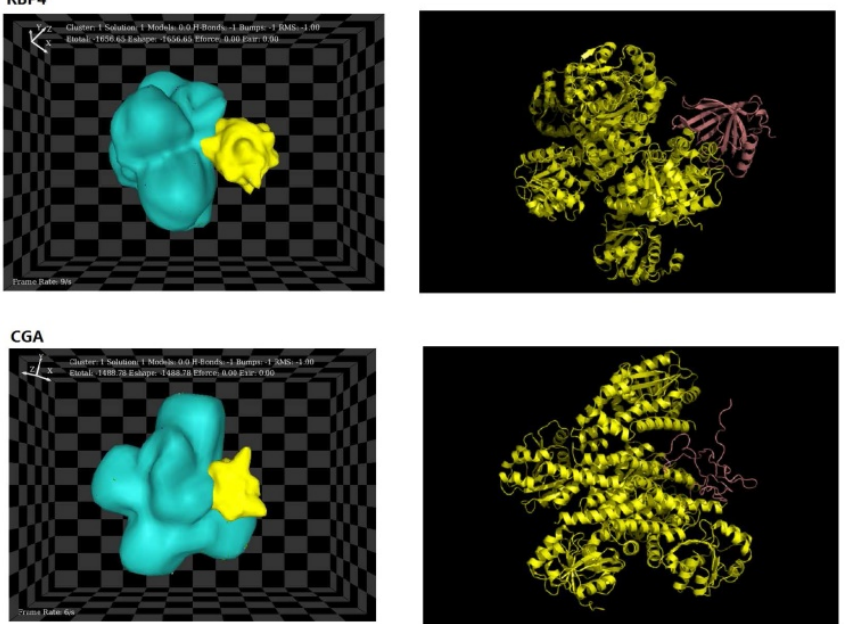
A
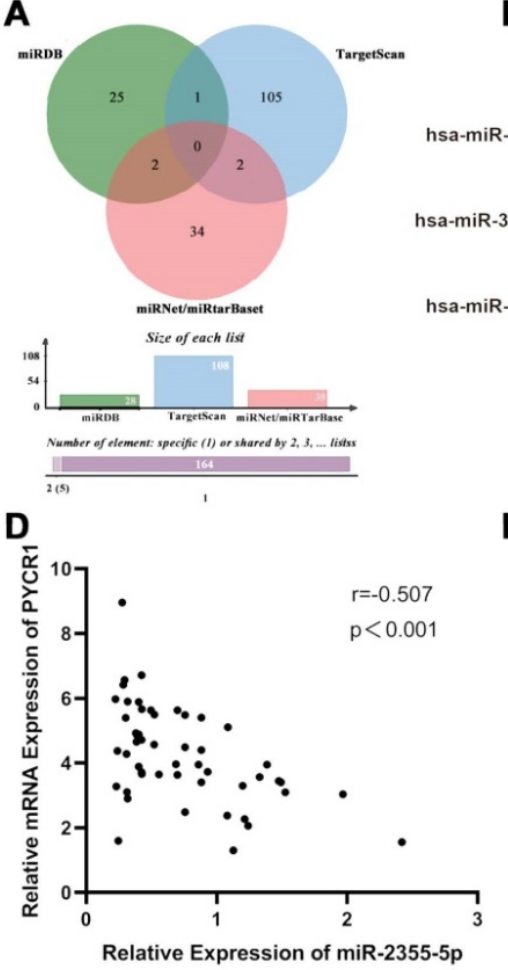

B

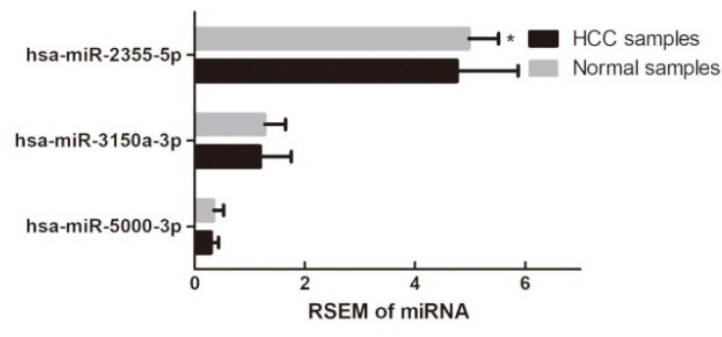

E

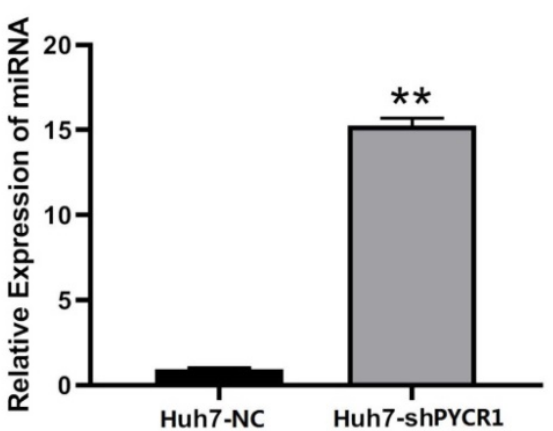

C

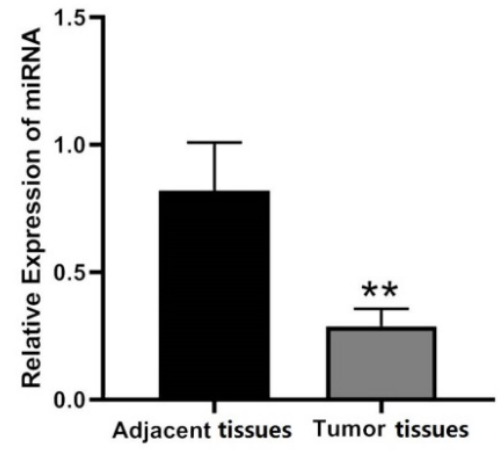

F

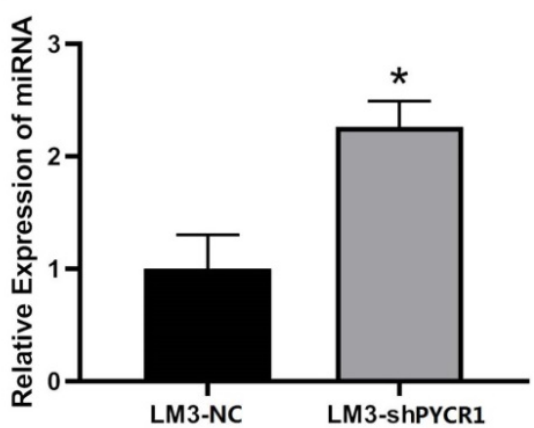

Figure 8. Potential miRNAs targeting PYCRI mRNA in HCC. (A) Online prediction of upstream regulatory miRNAs of PYCR1 mRNA; (B) Expression levels of miR-2355-5p, miR-3150a-3p, and miR-5000-3p in HCC and adjacent samples from TCGA; (C) Correlation analysis between PYCR1 mRNA and miR-2355-5p; (D) Validation of qRT-PCR: decreased miR-2355-5p in HCC tissues compared to adjacent tissues; (E, F) Up-regulated miR-2355-5p in HCC cell lines of Huh7 and LM3 after silencing PYCR1.

A previous study has shown that silencing of PYCR1 induces apoptosis and cell cycle arrest [1]; however, we did not obtain similar results. We employed lentivirus-mediated shRNAs to silence the expression of PYCR1 in Huh7 and LM3 HCC cells and to determine the effect of PYCR1 on various cellular functions. The results revealed that silencing PYCR1 induced a marked inhibition of cell proliferation, migration, and invasion. Further, the results of the HCC xenograft mouse study indicated that silencing PYCR1 significantly inhibited the tumor growth.

EMT has been shown to play an important role in tumorigenesis by inhibiting epithelial function and upregulating the expression of mesenchymal-related genes to enhance invasiveness and metastasis [44]. Moreover, epithelial cell characteristics and cell-cell adhesion were found to be disrupted, whereas migration and invasion properties were improved by EMT [45]. Specific markers of EMT (E-cadherin, $\mathrm{N}$-cadherin, vimentin, and $\beta$-catenin) were detected in our study. Cadherins are important factors in maintaining the stability of cell-cell adherens junctions and cellular signal transduction [44, 45]. Additionally, the expression of E-cadherin is decreased and that of $\mathrm{N}$-cadherin is increased in epithelial cells that are converted into fusoid mesenchymal cells during EMT. Furthermore, as one of the primary cytoskeletal components in mesenchymal cells, vimentin, whose concentration has been shown to increase during EMT, is closely associated with the differentiation, metastasis, and invasion of cancer cells [46]. $\beta$-catenin is a multifunctional protein, which, together with E-cadherin, serves a structural role in adherens junctions, thereby contributing to sustained unrestricted proliferation, survival, and metastatic spread of cancer cells [47]. In our study, the silencing of PYCR1 caused increased expression of E-cadherin and $\beta$-catenin and decreased expression of $\mathrm{N}$-cadherin and vimentin. These results suggest that PYCR1 promotes HCC migration and invasiveness by inducing EMT.

To better understand the downstream molecular events involved in the effects of PYCR1 on HCC invasiveness and metastasis, we performed RNA-seq to obtain DEG profiles following PYCR1 silencing. Two specific genes, namely, secreted phosphoprotein 1 (SPP1) and C-X-C motif chemokine 12 (CXCL12) were identified to be strongly associated with PYCR1. Previous studies have reported that PYCR1 can activate SPP1 to regulate cell adhesion [48, 49]. Furthermore, PYCR1 expression is regulated by the CXCL12SDF-1/CXCR7 signaling pathway, which regulates the expression of genes involved in cell cycle control, amino acid metabolism, and ligase activity $[50,51]$. The other DEGs that were identified in our 
study did not exhibit any obvious synergistic or antagonistic functions with PYCR1, and thus, their mechanisms in relation to PYCR1 require further elucidation.

The results of our functional analysis showed that the identified DEGs were primarily enriched in 14 functional categories, including cell communication, MAPK cascade, and cell migration. Cell communication is crucial for morphogenesis, cell differentiation, homeostasis, growth, and cell-cell interaction [52]. Downregulated histidine-rich glycoprotein (HRG), NR1H4, and transcription factor 21 (TCF21) are associated with cell communication and were identified to be induced by silencing PYCR1. Furthermore, HRG inhibits the tumor growth by facilitating clearance of dying tumor cells $[53,54]$; whereas NR1H4 deficiency has been shown to promote cell proliferation, inflammation, and tumorigenesis in the intestine [55, 56]. TCF21 is a specific class II basic helix-loop-helix transcription factor, which functions as a tumor suppressor, likely by inactivating the PI3K/AKT signaling pathway [57, 58].

A previous study has also suggested that p38 MAPK pathways may be activated by PYCR1 and by increased expression of its downstream targets that regulate cell proliferation and metastasis [59]. Another study found that the IRS1/JNK signaling pathway was significantly altered after PYCR1 silencing. IRS1 is a cytoplasmic substrate of the insulin receptor and insulin-like growth factor 1 receptor and plays prominent roles in human malignancies. Phosphorylation of IRS1 leads to the recruitment of downstream effectors and to the activation of the MAPK cascade, which promotes the activation of the PI3K cascade [23]. In this study, we identified specific genes involved in MAPK pathways, such as apolipoprotein A1 (APOA1) and Erb-B2 receptor tyrosine kinase 4 (ERBB4), which were differentially expressed following PYCR1 silencing. Silencing of PYCR1 could interfere cell proliferation through the alteration of IRS1/JNK signaling pathway which subsequently inhibited MAPK pathways.

Other studies have reported that APOA1 may inhibit the formation of tumor vessels and induce an antitumor immune microenvironment, thereby preventing tumor progression [60, 61]. Moreover, APOA1-induced apoptosis has been found to be closely related to the inhibition of the MAPK pathway [62]. It has also been reported that ERBB4 can enhance the proangiogenic potential via activation of the PI3K/AKT and MAPK/ERK pathways [63]. Furthermore, silencing of PYCR1 has been associated with the inhibition of proliferation and invasiveness of breast cancer cell lines via activation of the
AKT/ERK signaling pathway [35]. Additionally, caveolin (CAV)-1 and CAV2, which are involved in cell proliferation pathways, have been shown to be necessary for the control of E2-dependent cell growth [64]. Thus, downregulation of CAV1 results in increased cell proliferation; whereas downregulation of CAV2 promotes the growth of tumor cells $[65,66]$.

Many other functional categories were found to be associated with the DEGs identified in PYCR1-silenced cells. In particular, glycosylation, cell differentiation, homeostasis, cell adhesion, neuron/ nervous system, cell component, and immune stimulus categories were identified. Taken together these results suggest that downregulation of PYCR1 induces the activation of these functional pathways, which may subsequently influence the development of HCC.

The results of our molecular docking analysis suggest that PYCR1 binds to its interacting proteins via binding hotspots. In the MAPK cascade, the DUSP9-PYCR1 complex exhibited the lowest $E_{\text {total }}$ value (-906.94 $\mathrm{kcal} \mathrm{mol}^{-1}$ ) compared with those of two other complexes (APOA1-PYCR1 and GRIK2PYCR1). Further, complexes of PYCR1 with proteins that are most strongly related to cell migration and cell communication exhibited the lowest $\mathrm{E}_{\text {total }}$ values, namely, SERPINE1-PYCR1 $\left(-1,889.79 \mathrm{kcal}^{\mathrm{mol}}{ }^{-1}\right)$ and NR1H4-PYCR1 $\left(-1,659.98 \quad \mathrm{kcal} \mathrm{mol}^{-1}\right)$. Previous research has suggested that lower $E_{\text {total }}$ values are associated with easier and stronger binding between a ligand and a receptor [31]. Therefore, our results suggest that functional binding between PYCR1 and DUSP9, SERPINE1, and NR1H4 may mediate the MAPK cascade, cell migration, and cell communication, respectively.

Analysis of hotspots revealed that several amino acid residues in DUSP9 and SERPINE1 exclusively contributed to PYCR1 binding, whereas no hotspots were identified in other protein-protein complexes involved in the MAPK cascade, cell migration, and cell-cell communication. Moreover, several hotspots in the CGA-PYCR1 complex were found to be involved in cell proliferation and growth. These results suggest that the CGA-PYCR1 complex is multifunctional. Interestingly, all of the identified PYCR1 hotspots included Arg, which may imply that Arg is vital for the binding between PYCR1 and its interacting proteins. It has been reported that the depletion of Arg may suppress the proliferation of HCC cells because reduced Arg levels would interfere with intracellular glutamine metabolism and inhibit the synthesis of proteins and thymidine $[67,68]$. Since our results indicate that Arg may play an important role in the binding between PYCR1 and its interacting proteins, Arg may serve as a potential target for HCC 
treatment.

Various studies have demonstrated that noncoding RNAs participate in HCC development [69]. As a family of small and evolutionarily conserved noncoding RNAs, miRNAs are capable of regulating physiological and pathological processes by inhibiting target mRNA translation or promoting mRNA degradation [70]. Our in silico prediction revealed that miR-2355-5p might be an upstream regulator for PYCR1 mRNA. Importantly, when PYCR1 levels were upregulated, miR-2355-5p was downregulated in HCC tissues. These results indicated that miR-2355-5p could serve as a negative regulator of PYCR1 expression, and low miR-2355-5p expression could promote the accumulation of PYCR1 in HCC tissues. Furthermore, miR-2355-5p has been reported to be a master regulator of target genes in other diseases, including chondrosarcoma and intervertebral disc degeneration [71, 72]. However, the specific mechanism underlying downregulation of miR-2355-5p in HCC remains unclear.

In summary, our results suggest a role of PYCR1 in inducing the development of HCC. PYCR1 was found to be upregulated in HCC tissues compared to paired adjacent nontumor tissues. Using a TCGA dataset, we found that patients with low PYCR1 expression exhibited a higher OS rate than in patients with high PYCR1 expression. Meanwhile, higher PYCR1 expression was observed in females than in males and correlated with elevated AFP levels, higher clinical staging (stages III and IV), and a younger age (< 45 years old). In Chinese patients, two features, namely, higher clinical staging (stages III and IV) and a younger age ( $<45$ years old), were related to higher PYCR1 expression. When PYCR1 expression was silenced, the proliferation, migration, invasion, metastasis, and EMT, as well as the tumorigenic capacity, were significantly inhibited in HCC cells. The mechanisms responsible for these effects involved many proteins, which were associated with many functional categories and signaling pathways. Analysis of protein-protein interactions indicated that specific hotspots might facilitate the formation of HCC-specific PYCR1 complexes and promote the development of HCC. We therefore propose that PYCR1 may be an effective novel target for the development of diagnostic or therapeutic options for HCC.

\section{Supplementary Material}

Supplementary figures and tables.

http://www.ijbs.com/v17p2223s1.pdf

\section{Acknowledgements}

This work was supported by the Guangxi
Natural Science Foundation (grant numbers: GuikeAB17195006, 2018GXNSFAA281033, 2016GXNSFDA380010, 2016GXNSFBA380016, and 2018GXNSFBA138020); the National Natural Science Foundation of China (grant numbers: 81660460 and 81660470); and the Guangxi Key Laboratory of Bio-targeted Diagnosis and Treatment Research Open Fund (grant number: GXSWBX201805).

\section{Data availability}

RNA-seq data have been deposited into the National Center for Biotechnology Information GEO public database under the accession code GSE171983.

\section{Competing Interests}

The authors have declared that no competing interest exists.

\section{References}

1. Zeng $\mathrm{T}$, Zhu L, Liao M, et al. Knockdown of PYCR1 inhibits cell proliferation and colony formation via cell cycle arrest and apoptosis in prostate cancer. Med Oncol. 2017; 34: 27.

2. Liu W, Phang JM. Proline dehydrogenase (oxidase) in cancer. Biofactors. 2012; 38: 398-406.

3. Tanner JJ, Fendt SM, Becker DF. The Proline Cycle As a Potential Cancer Therapy Target. Biochemistry. 2018; 57: 3433-3444.

4. Liu W, Hancock CN, Fischer JW, et al. Proline biosynthesis augments tumor cell growth and aerobic glycolysis: involvement of pyridine nucleotides. Sci Rep. 2015; 5: 17206.

5. Phang JM, Yeh GC, Hagedorn $\mathrm{CH}$. The intercellular proline cycle. Life Sci. $1981 ; 28:$ 53-58.

6. Hagedorn $\mathrm{CH}$, Phang JM. Transfer of reducing equivalents into mitochondria by the interconversions of proline and delta 1-pyrroline-5-carboxylate. Arch Biochem Biophys. 1983; 225: 95-101.

7. Fischer-Zirnsak B, Escande-Beillard N, Ganesh J, et al. Recurrent De Novo Mutations Affecting Residue Arg138 of Pyrroline-5-Carboxylate Synthase Cause a Progeroid Form of Autosomal-Dominant Cutis Laxa. Am J Hum Genet. 2015; 97: 483-492.

8. Abul Seoud RA, Mabrouk MS. TMT-HCC: a tool for text mining the biomedical literature for hepatocellular carcinoma (HCC) biomarkers identification. Comput Methods Programs Biomed. 2013; 112: 640-648.

9. Pang TC, Lam VW. Surgical management of hepatocellular carcinoma. World J Hepatol. 2015; 7: 245-252.

10. Petruzziello A, Marigliano S, Loquercio G, et al. Global epidemiology of hepatitis $C$ virus infection: An up-date of the distribution and circulation of hepatitis C virus genotypes. World J Gastroenterol. 2016; 22: 7824-7840.

11. Wu J, Yang S, Xu K, et al. Patterns and Trends of Liver Cancer Incidence Rates in Eastern and Southeastern Asian Countries (1983-2007) and Predictions to 2030. Gastroenterology. 2018; 154: 1719-1728.e1715.

12. Kucukcakan B, Hayrulai-Musliu Z. Challenging Role of Dietary Aflatoxin B1 Exposure and Hepatitis B Infection on Risk of Hepatocellular Carcinoma. Open Access Maced J Med Sci. 2015; 3: 363-369.

13. Finn RS. Emerging targeted strategies in advanced hepatocellular carcinoma. Semin Liver Dis. 2013; 33 Suppl 1: S11-19.

14. Cabrera R, Nelson DR. Review article: the management of hepatocellular carcinoma. Aliment Pharmacol Ther. 2010; 31: 461-476.

15. Wang M, Yu F, Li P. Circular RNAs: Characteristics, Function and Clinical Significance in Hepatocellular Carcinoma. Cancers (Basel). 2018; 10.

16. $\mathrm{Yu}$ SJ. A concise review of updated guidelines regarding the management of hepatocellular carcinoma around the world: 2010-2016. Clin Mol Hepatol. 2016; 22: 7-17.

17. Petrick JL, Braunlin M, Laversanne $M$, et al. International trends in liver cancer incidence, overall and by histologic subtype, 1978-2007. Int J Cancer. 2016; 139: 1534-1545.

18. Cai F, Miao Y, Liu C, et al. Pyrroline-5-carboxylate reductase 1 promotes proliferation and inhibits apoptosis in non-small cell lung cancer. Oncol Lett. 2018; 15: 731-740. 
19. Ernst $\mathrm{T}$, Hergenhahn $\mathrm{M}$, Kenzelmann $\mathrm{M}$, et al. Decrease and gain of gene expression are equally discriminatory markers for prostate carcinoma: a gene expression analysis on total and microdissected prostate tissue. Am J Pathol. 2002; 160: 2169-2180.

20. Possemato R, Marks KM, Shaul YD, et al. Functional genomics reveal that the serine synthesis pathway is essential in breast cancer. Nature. 2011; 476: 346-350.

21. Febbo PG, Sellers WR. Use of expression analysis to predict outcome after radical prostatectomy. J Urol. 2003; 170: S11-19; discussion S19-20.

22. Craze ML, Cheung $\mathrm{H}$, Jewa $\mathrm{N}$, et al. MYC regulation of glutamine-proline regulatory axis is key in luminal B breast cancer. $\mathrm{Br} \mathrm{J}$ Cancer. 2018; 118: 258-265.

23. Zhuang J, Song Y, Ye Y, et al. PYCR1 interference inhibits cell growth and survival via c-Jun $\mathrm{N}$-terminal kinase/insulin receptor substrate 1 (JNK/IRS1) pathway in hepatocellular cancer. J Transl Med. 2019; 17: 343.

24. Chin MH, Mason MJ, Xie W, et al. Induced pluripotent stem cells and embryonic stem cells are distinguished by gene expression signatures. Cell Stem Cell. 2009; 5: 111-123.

25. Liu K, Zhang Y, Zhang C, et al. Methylation of S100A8 is a promising diagnosis and prognostic marker in hepatocellular carcinoma. Oncotarget. 2016; 7: 56798-56810.

26. Jia W, Xie L, Wang X, et al. The impact of MCM6 on hepatocellular carcinoma in a Southern Chinese Zhuang population. Biomed Pharmacother. 2020; 127: 110171.

27. Liu JY, Dong $\mathrm{XX}, \mathrm{Lu} \mathrm{JN}$, et al. Utility of GDF-15 as a diagnostic biomarker in gastric cancer: an investigation combining GEO, TCGA and meta-analysis. FEBS Open Bio. 2019; 9: 35-42.

28. Liang HW, Yang X, Wen DY, et al. Utility of miR-133a-3p as a diagnostic indicator for hepatocellular carcinoma: An investigation combined with GEO, TCGA, meta-analysis and bioinformatics. Mol Med Rep. 2018; 17: 1469-1484.

29. Li J, Dou D, Li P, et al. PARP-1 serves as a novel molecular marker for hepatocellular carcinoma in a Southern Chinese Zhuang population. Tumour Biol. 2017; 39: 1010428317706914.

30. Schmittgen TD, Livak KJ. Analyzing real-time PCR data by the comparative C(T) method. Nat Protoc. 2008; 3: 1101-1108.

31. Kim D, Langmead B, Salzberg SL. HISAT: a fast spliced aligner with low memory requirements. Nat Methods. 2015; 12: 357-360.

32. Li H, Handsaker B, Wysoker A, et al. The Sequence Alignment/Map format and SAMtools. Bioinformatics. 2009; 25: 2078-2079.

33. Liao Y, Smyth GK, Shi W. featureCounts: an efficient general purpose program for assigning sequence reads to genomic features. Bioinformatics. 2014; 30: 923-930.

34. Love MI, Huber W, Anders S. Moderated estimation of fold change and dispersion for RNA-seq data with DESeq2. Genome Biol. 2014; 15: 550.

35. Liu Q, Yang J, Cai J, et al. Analysis of the Interactions Between Thioredoxin and 20 Selenoproteins in Chicken. Biol Trace Elem Res. 2017; 179: 304-317.

36. Denis B, Grubier M, Avezou FC, et al. [Rupture of the tricuspid cordae simulating a right intraventricular tumor]. Arch Mal Coeur Vaiss. 1975; 68: 887-891.

37. Guo L, Cui C, Zhang K, et al. Kindlin-2 links mechano-environment to proline synthesis and tumor growth. Nat Commun. 2019; 10: 845.

38. Kuo ML, Lee MB, Tang M, et al. PYCR1 and PYCR2 Interact and Collaborate with RRM2B to Protect Cells from Overt Oxidative Stress. Sci Rep. 2016; 6: 18846

39. Chen S, Yang X, Yu M, et al. SIRT3 regulates cancer cell proliferation through deacetylation of PYCR1 in proline metabolism. Neoplasia. 2019; 21: 665-675.

40. Yan $\mathrm{K}, \mathrm{Xu} \mathrm{X}, \mathrm{Wu} \mathrm{T}$, et al. Knockdown of PYCR1 inhibits proliferation, drug resistance and EMT in colorectal cancer cells by regulating STAT3-Mediated p38 MAPK and NF-kB signalling pathway. Biochem Biophys Res Commun. 2019; 520: 486-491.

41. Ding J, Kuo ML, Su L, et al. Human mitochondrial pyrroline-5-carboxylate reductase 1 promotes invasiveness and impacts survival in breast cancers. Carcinogenesis. 2017; 38: 519-531.

42. Yasuda T, Kaji Y, Agatsuma T, et al. DJ-1 cooperates with PYCR1 in cell protection against oxidative stress. Biochem Biophys Res Commun. 2013; 436: 289-294.

43. Gao P, Tchernyshyov I, Chang TC, et al. c-Myc suppression of miR-23a/b enhances mitochondrial glutaminase expression and glutamine metabolism. Nature. 2009; 458: 762-765.

44. Giri AK, Midha S, Banerjee P, et al. Common Variants in CLDN2 and MORC4 Genes Confer Disease Susceptibility in Patients with Chronic Pancreatitis. PLoS One. 2016; 11: e0147345.

45. Thiery JP, Acloque H, Huang RY, et al. Epithelial-mesenchymal transitions in development and disease. Cell. 2009; 139: 871-890.
46. Li F, Dai L, Niu J. GPX2 silencing relieves epithelial-mesenchymal transition, invasion, and metastasis in pancreatic cancer by downregulating Wnt pathway. J Cell Physiol. 2020; 235: 7780-7790.

47. $\mathrm{Xu} \mathrm{X}$, Liu Z, Wang J, et al. Global proteomic profiling in multistep hepatocarcinogenesis and identification of PARP1 as a novel molecular marker in hepatocellular carcinoma. Oncotarget. 2016; 7: 13730-13741.

48. Sun Y, Wang L, Jiang M, et al. Secreted phosphoprotein 1 upstream invasive network construction and analysis of lung adenocarcinoma compared with human normal adjacent tissues by integrative biocomputation. Cell Biochem Biophys. 2010; 56: 59-71.

49. Li Y, Du W, Han J, et al. LAMP3 promotes the invasion of osteosarcoma cells via SPP1 signaling. Mol Med Rep. 2017; 16: 5947-5953.

50. Yoshida D, Nomura R, Teramoto A. Signalling pathway mediated by CXCR7, an alternative chemokine receptor for stromal-cell derived factor-1a, in AtT20 mouse adrenocorticotrophic hormone-secreting pituitary adenoma cells. J Neuroendocrinol. 2009; 21: 481-488.

51. $\mathrm{Yu} \mathrm{PF}$, Huang $\mathrm{Y}, \mathrm{Xu} \mathrm{CL}$, et al. Downregulation of CXCL12 in mesenchymal stromal cells by TGF $\beta$ promotes breast cancer metastasis. Oncogene. 2017; 36: 840-849.

52. Brücher BL, Jamall IS. Cell-cell communication in the tumor microenvironment, carcinogenesis, and anticancer treatment. Cell Physiol Biochem. 2014; 34: 213-243.

53. Li Z, Lv T, Liu Y, et al. PARP1 is a novel independent prognostic factor for the poor prognosis of chordoma. Cancer Biomark. 2016; 16: 633-639.

54. Kärrlander M, Lindberg N, Olofsson T, et al. Histidine-rich glycoprotein can prevent development of mouse experimental glioblastoma. PLoS One. 2009; 4: e8536

55. Sharma PM, Ponnaiya B, Taveras $M$, et al. High throughput measurement of $\gamma \mathrm{H} 2 \mathrm{AX}$ DSB repair kinetics in a healthy human population. PLoS One. 2015; 10: e0121083.

56. Maran RR, Thomas A, Roth M, et al. Farnesoid X receptor deficiency in mice leads to increased intestinal epithelial cell proliferation and tumor development. J Pharmacol Exp Ther. 2009; 328: 469-477.

57. Chen B, Zeng C, Ye Y, et al. Promoter methylation of TCF21 may repress autophagy in the progression of lung cancer. J Cell Commun Signal. 2018; 12: 423-432.

58. Dai Y, Duan H, Duan C, et al. TCF21 functions as a tumor suppressor in colorectal cancer through inactivation of $\mathrm{PI} 3 \mathrm{~K} / \mathrm{AKT}$ signaling. Onco Targets Ther. 2017; 10: 1603-1611.

59. Wang D, Wang L, Zhang Y, et al. PYCR1 promotes the progression of non-small-cell lung cancer under the negative regulation of miR-488. Biomed Pharmacother. 2019; 111: 588-595.

60. Gao F, Vasquez SX, Su F, et al. L-5F, an apolipoprotein A-I mimetic, inhibits tumor angiogenesis by suppressing VEGF/basic FGF signaling pathways. Integr Biol (Camb). 2011; 3: 479-489.

61. Rouleau M, Patel A, Hendzel MJ, et al. PARP inhibition: PARP1 and beyond. Nat Rev Cancer. 2010; 10: 293-301.

62. Wei $\mathrm{H}, \mathrm{Yu} X$. Functions of PARylation in DNA Damage Repair Pathways. Genomics Proteomics Bioinformatics. 2016; 14: 131-139.

63. Kim J, Yum S, Kang C, et al. Gene-gene interactions in gastrointestinal cancer susceptibility. Oncotarget. 2016; 7: 67612-67625.

64. Totta P, Gionfra F, Busonero C, et al. Modulation of $17 \beta$-Estradiol Signaling on Cellular Proliferation by Caveolin-2. J Cell Physiol. 2016; 231: 1219-1225.

65. Liu F, Shangli Z, Hu Z. CAV2 promotes the growth of renal cell carcinoma through the EGFR/PI3K/Akt pathway. Onco Targets Ther. 2018; 11: 6209-6216.

66. Arshad M, Conzelmann C, Riaz MA, et al. Inhibition of Cx43 attenuates ERK1/2 activation, enhances the expression of Cav-1 and suppresses cell proliferation. Int J Mol Med. 2018; 42: 2811-2818.

67. Dabrowska K, Skowronska K, Popek M, et al. Roles of Glutamate and Glutamine Transport in Ammonia Neurotoxicity: State of the Art and Question Marks. Endocr Metab Immune Disord Drug Targets. 2018; 18 : 306-315.

68. Hecker M, Sessa WC, Harris HJ, et al. The metabolism of L-arginine and its significance for the biosynthesis of endothelium-derived relaxing factor: cultured endothelial cells recycle L-citrulline to L-arginine. Proc Natl Acad Sci U S A. 1990; 87: 8612-8616.

69. Heo MJ, Yun J, Kim SG. Role of non-coding RNAs in liver disease progression to hepatocellular carcinoma. Arch Pharm Res. 2019; 42: 48-62.

70. Huang $\mathrm{Y}$. The novel regulatory role of lncRNA-miRNA-mRNA axis in cardiovascular diseases. J Cell Mol Med. 2018; 22: 5768-5775.

71. Cheng $C$, Zhang $Z$, Cheng $F$, et al. Exosomal IncRNA RAMP2-AS1 Derived from Chondrosarcoma Cells Promotes Angiogenesis Through miR-2355-5p/VEGFR2 Axis. Onco Targets Ther. 2020; 13: 3291-3301.

72. Guo $Y$, Tian $L$, Liu $X$, et al. ERRFI1 Inhibits Proliferation and Inflammation of Nucleus Pulposus and Is Negatively Regulated by 
Int. J. Biol. Sci. 2021, Vol. 17

miR-2355-5p in Intervertebral Disc Degeneration. Spine (Phila Pa 1976). 2019; 44: E873-e881. 